\title{
Adaptive Dynamic Surface Decentralized Output Feedback Control for Switched Nonstrict Feedback Large-Scale Systems with Unknown Dead Zones
}

\author{
Min Wan (i) and Shanshan Huang (D) \\ School of Mechatronic Engineering, Southwest Petroleum University, Chengdu, China \\ Correspondence should be addressed to Min Wan; 18940103@qq.com
}

Received 5 April 2020; Revised 18 July 2020; Accepted 20 August 2020; Published 27 September 2020

Academic Editor: Jose Vicente Salcedo

Copyright (C) 2020 Min Wan and Shanshan Huang. This is an open access article distributed under the Creative Commons Attribution License, which permits unrestricted use, distribution, and reproduction in any medium, provided the original work is properly cited.

\begin{abstract}
This paper investigates a novel adaptive output feedback decentralized control scheme for switched nonstrict feedback large-scale systems with unknown dead zones. A decentralized linear state observer is designed to estimate the unmeasurable states of subsystems. The dead zone inverse technique is used to compensate the effect of the unknown dead zone. A variable separation approach is applied to deal with the nonstrict feedback problem. Moreover, dynamic surface control and minimal parameter learning technology are adopted to reduce the computation burden. The proof of stability and the arbitrary switching are obtained by the common Lyapunov method. Finally, simulation results are given to show the effectiveness of the proposed control scheme.
\end{abstract}

\section{Introduction}

With the development of science and technology, the complexity of control plant is increasing, and more and more large-scale interconnected systems appear. It is well known that the decentralized control can reduce the computational burden compared with the centralized control strategy. Therefore, as an effective control scheme, the decentralized control has been widely used for large-scale nonlinear system control. Jain and Khorrami first discussed the decentralized adaptive control of a class of large-scale interconnected nonlinear systems in [1], which did not require the unknown interconnected terms of the system to meet the so-called matching conditions. Sun et al. [2] studied the fuzzy adaptive decentralized optimal control problem of the strict feedback large-scale system. Yoo and Park[3] proposed an adaptive decentralized output feedback control method for a large-scale system with unknown time-varying delayed interactions. In [4], a decentralized adaptive output feedback controller was designed for a stochastic nonlinear interconnected system. In [5], based on backstepping technology, an adaptive decentralized neural $H_{\infty}$ tracking control scheme was proposed for a class of large-scale interconnected systems. In [6], to deal with the output feedback control problem of the stochastic large-scale system with unknown dead zone, an adaptive fuzzy decentralized control method based on state observer was proposed. In [7], a decentralized control scheme was discussed for a SISO large-scale system with strong interconnection. Huang and Wang [8] extended the results in [7] to control a class of large-scale MIMO systems with strong interconnection and successfully applied the control method to the automated highway systems.

The aforementioned results all focus on large-scale uncertain nonlinear systems. In the actual engineering, a lot of systems not only contain many low-dimensional subsystems but also have switched properties. That is to say, these systems are not only large-scale interconnected systems but also switched systems. For example, the robot cooperative control system, spacecraft control system, power system, large-scale traffic control system, communication system, and so on are all switched large-scale systems. Recently, the control problem of large-scale interconnected switched nonlinear systems has aroused the researchers' 
strong interest, and a small number of relevant results have appeared. Wang and Tong [9] studied the adaptive output feedback control problem for a class of switched Takagi-Sugeno fuzzy large-scale systems with unmeasured states. $\mathrm{Li}$ and Tong [10] investigated the adaptive fuzzy output feedback control problem of a switched large-scale system with nonstrict feedback structure. In [11], by adopting switched Lyapunov function with $H_{\infty}$ performance, the decentralized control was designed for switched fuzzy largescale Takagi-Sugeno systems. However, there are no results on the control problems of large-scale switched nonstrict feedback systems with input constraints.

Many actuators used for industrial control, such as the servo motor, reducer, gear, hydraulic proportional servo system, cylinder, and so on, have input constraints due to mechanical structure design, manufacturing, and friction damping $[12,13]$. Unknown dead zone will seriously affect the control performance of the control system, such as reduce the control accuracy, or even completely fail or produce safety accident caused by self-excited oscillation. In recent years, in order to solve the control problem caused by the dead zone of actuators, some researchers in the field of control have carried out more in-depth research and achieved some research results. In [14], an adaptive fuzzy tracking control was designed for a class of pure feedback nonlinear systems with unknown dead zone. In [15], based on backstepping control, a fuzzy output feedback tracking control method was proposed for the strict feedback system with unknown dead zone. In [16], by using dynamic surface control, an adaptive control problem was studied for a pure feedback system with unknown dead zone. Wang and Chen [17] studied the problem of adaptive fuzzy dynamic surface control of nonstrict feedback nonlinear systems with unknown virtual control coefficients, dead zone, and full state constraints. However, none of the above results have considered the control problem of switched systems and switched large-scale systems. In [18], Tong et al. proposed an adaptive fuzzy decentralized tracking control scheme based on state observer for switched large-scale systems with unknown dead zone. But the system in [18] was strict feedback form, and there existed the problem of "explosion of complexity."

This paper will study the control problem of large-scale switched nonlinear systems with nonstrict feedback structure and use the common Lyapunov function method to overcome the arbitrary switching problem. Moreover, adaptive parameters will be designed to identify the unknown dead zone parameters through dead zone inverse technology. Compared with the previous literature, the main advantages of the proposed control scheme are listed as follows:

(1) For the first time, the control problem of large-scale switched systems with nonstrict feedback structure and unknown dead zones is studied. The proposed control scheme is quite different from the existing results. The control method in [19-24] cannot be used to control switched systems. In [25-28], the control problem of switched systems was studied, whereas these methods cannot control switched large-scale systems. Moreover, unknown dead zone was not considered. Though switched large-scale systems with dead zones were considered in $[18,29]$, they were strict feedback form, rather than nonstrict feedback system in this paper. The system in [10] was a switched large-scale nonstrict feedback system, but it was without a dead zone, and the control method had the drawback of "explosion of complexity."

(2) The control method proposed in this paper does not need $n$ order differentiable and bounded conditions of input signals and a monotonically increasing condition of unknown functions. However, these strict assumptions are common in the existing literature $[30,31]$. Because of using dynamic surface control, "explosion of complexity" problem is avoided in this paper. Moreover, by applying linear state observer and minimal parameter learning technology, the proposed control strategy only contains $6 M$ online learning parameters for the switched large-scale system, where $M$ is the number of interconnected subsystems. Therefore, the computation burden is reduced significantly.

(3) The results in this paper are developed with full consideration of the dead zone problem. By constructing a dead zone compensator, the proposed adaptive fuzzy decentralized output feedback control approach can overcome the unknown dead zone problem of uncertain switched nonlinear large-scale nonstrict feedback systems.

\section{Problem Description and Preliminaries}

The switched nonstrict feedback large-scale system considered in this paper has $M$ interconnected subsystems. $k t h$ subsystem $\sum_{k}(k=1,2, \ldots, M)$ is shown as follows [10]:

$$
\left\{\begin{array}{l}
\dot{x}_{k, 1}=x_{k, 2}+f_{k, 1}^{\sigma(t)}\left(\underline{x}_{k}\right)+h_{k, 1}^{\sigma(t)}(\underline{y}), \\
\dot{x}_{k, 2}=x_{k, 3}+f_{k, 2}^{\sigma(t)}\left(\underline{x}_{k}\right)+h_{k, 2}^{\sigma(t)}(\underline{y}), \\
\vdots \\
\dot{x}_{k, m_{k}-1}=x_{k, m_{k}}+f_{k, m_{k}-1}^{\sigma(t)}\left(\underline{x}_{k}\right)+h_{k, m_{k}-1}^{\sigma(t)}(\underline{y}), \\
\dot{x}_{k, m_{k}}=D_{k} u_{k}+f_{k, m_{k}}^{\sigma(t)}\left(\underline{x}_{k}\right)+h_{k, m_{k}}^{\sigma(t)}(\underline{y}), \\
y_{k}=x_{k, 1},
\end{array}\right.
$$

where $\underline{x}_{k}=\left[x_{k, 1}, x_{k, 2}, \ldots, x_{k, m_{k}}\right]^{\mathrm{T}} \in R^{m_{k}}$ is the state vector of $\sum_{k}$, and only $x_{k, 1}$ can be measured. $y=\left[y_{1}, \ldots, y_{M}\right] \in R^{M}$ is the output vector of the large-scale system. $f_{k, l}^{\sigma(t)}\left(\underline{x}_{k}\right)$ and $h_{k, l}^{\sigma(t)}(y)\left(1 \leq k \leq M, 1 \leq l \leq m_{k}\right)$ are unknown smooth functions, and $h_{k, l}^{\sigma(t)}(y)$ represents the coupling effect between $M$ subsystems of the large-scale system. $\sigma(t):[0, \infty) \longrightarrow N=$ $\{1,2, \ldots, n\}$ is the switching signal, which is a piecewise continuous function of time from the right. The sth subsystem is active when $\sigma(t)=s . u_{k} \in R$ is the actual control input of $\sum_{k}$. $D_{k} u_{k} \in R$ is the output of the unknown dead zone with input $u_{k}$, which can represented as [32] 


$$
D_{k} u_{k}= \begin{cases}m_{k, r}\left(u_{k}-d_{k, r}\right), & u_{k} \geq d_{k, r}, \\ 0, & -d_{k, l}<u_{k}<d_{k, r}, \\ m_{k, l}\left(u_{k}+d_{k, l}\right), & u_{k} \leq-d_{k, l},\end{cases}
$$

where $m_{k, r}$ and $m_{k, l}$ denote the unknown slope of the dead zone and $d_{k, l}$ and $d_{k, r}$ denote the unknown width parameters of the dead zone.

Assumption 1 (see $[9,10]$ ). For $1 \leq k \leq M$ and $1 \leq l \leq m_{k}$, there exists unknown smooth function $\hbar_{k, l, i}^{s}\left(y_{i}\right)$, such that

$$
\left|h_{k, l}^{s}(\underline{y})\right|^{2} \leq \sum_{i=1}^{M}\left(y_{i} \hbar_{k, l, i}^{s}\left(y_{i}\right)\right)^{2}
$$

where $s \in N$.

Assumption 2 (see $[9,10])$. There exists unknown smooth function $\bar{f}_{k, l}^{s}\left(\underline{x}_{k}\right)$, such that $f_{k, l}^{s}\left(\underline{x}_{k}\right)=x_{k, 1} \bar{f}_{k, l}^{s}\left(\underline{x}_{k}\right)$, where $l=1,2, \ldots, m_{k}$.

Assumption 3. The reference signal $y_{k, d}(t)$ and its first derivative exist and are bounded.

Assumption 4 (see [32]). The output of dead zone $D_{k} u_{k} \in R$ is unmeasurable, and the parameters $m_{k, r}, m_{k, l}, d_{k, l}$, and $d_{k, r}$ are unknown, whereas the signs are known such that $m_{k, r}>0, m_{k, l}>0, d_{k, l} \geq 0$, and $d_{k, r} \geq 0$, respectively.

Assumption 5 (see [32]). The slopes of the dead zone are bounded by constants $m_{k, r \text { min }}, m_{k, r \text { max }}, m_{k, l \text { min }}$, and $m_{k, l \text { max }}$ and satisfy $0<m_{k, r \min } \leq m_{k, r} \leq m_{k, r \max }$ and $0<m_{k, l \text { min }} \leq$ $m_{k, l} \leq m_{k, l \text { max }}$.

Remark 1. It should be mentioned that if for $t \in[0, \infty)$, the switching signal $\sigma(t)=s$, then system (1) represents a class of nonswitched nonlinear large-scale systems in nonstrict feedback form, which is investigated commonly in the existing literature.

Remark 2. It is worth pointing out that the switched systems are different from those nonswitched systems, which brings more difficulties in the control design of switched systems than those of nonswitched systems.

In this paper, the dead zone inverse technique will be used to compensate the unknown dead zone effect. Defining $u_{k, d} \in R$ as the control input from the controller to achieve the control objective for the plant without a dead zone, $u_{k} \in R$ can be generated according to the certainty equivalence dead zone inverse $[29,32]$ :

$$
u_{k}=D_{k}^{-1}\left(u_{k, d}\right)=\frac{u_{k, d}+\widehat{d}_{k, m r}}{\widehat{m}_{k, r}} \delta_{k}+\frac{u_{k, d}-\widehat{d}_{k, m l}}{\widehat{m}_{k, l}}\left(1-\delta_{k}\right),
$$

where $\widehat{m}_{k, r}, \hat{m}_{k, l}, \hat{d}_{k, m r}$, and $\hat{d}_{k, m l}$ are the estimations of $m_{k, r}$, $m_{k, l}, m_{k, r} d_{k, r}$, and $m_{k, l} d_{k, l}$, respectively. Moreover, $\delta_{k}$ satisfies

$$
\delta_{k}= \begin{cases}1, & u_{k, d} \geq 0 \\ 0, & u_{k, d}<0\end{cases}
$$

Then, we can have the error between $D_{k}\left(u_{k}\right)$ and $u_{k, d}$ :

$$
\begin{aligned}
D_{k}\left(u_{k}\right)-u_{k, d}= & \left(\tilde{d}_{k, m r}-\frac{u_{k, d}+\widehat{d}_{k, m r}}{\hat{m}_{k, r}} \widetilde{m}_{k, r}\right) \delta_{k} \\
& +\left(-\widetilde{d}_{k, m l}-\frac{u_{k, d}-\widehat{d}_{k, m l}}{\hat{m}_{k, l}} \widetilde{m}_{k, l}\right)\left(1-\delta_{k}\right)+\varepsilon_{k, d},
\end{aligned}
$$

where $\quad \widetilde{m}_{k, r}=\widehat{m}_{k, x}-m_{k, r 2}, \quad \widetilde{m}_{k, l}=\widehat{m}_{k, l}-m_{k, l}, \quad \tilde{d}_{k, m r}=$ $\widehat{d}_{k, m r}-d_{k, m r}$, and $d_{k, m l}=\hat{d}_{k, m l}-d_{k, m l}$ are the parameter estimate errors; $\varepsilon_{k, d}=-m_{k, r} \chi_{k, r}\left(u_{k}-d_{k, r}\right)-m_{k, l} \chi_{k, l}\left(u_{k}+d_{k, l}\right)$ is bounded, where $\chi_{k, r}$ and $\chi_{k, l}$ are as follows:

$$
\begin{aligned}
\chi_{k, r} & = \begin{cases}1, & 0 \leq u_{k}<d_{k, r}, \\
0, & u_{k}<0, u_{k} \geq d_{k, r},\end{cases} \\
\chi_{k, l} & = \begin{cases}1, & -d_{k, l} \leq u_{k}<0, \\
0, & u_{k}<-d_{k, l}, u_{k} \geq 0 .\end{cases}
\end{aligned}
$$

Control Objective. The control objective is to design an adaptive output feedback decentralized control scheme to keep all the outputs $y_{k}(t)$ of the subsystem tracking the desired trajectories $y_{k, d}(t)$ under unknown dead zones, respectively. Moreover, the tracking errors can be kept as small as possible and all the signals of the closed system are bounded.

\section{The Approximation of Fuzzy Logic Systems}

A fuzzy logic system can be written as $\hat{f}(x \mid \theta)=\theta^{T} \xi(x)$, where $\xi(x)$ is the fuzzy basis function vector and $\theta \in R^{N}$ is the adjustable weight parameter vector.

Lemma 1 (see [33-35]). If $f(x)$ is a continuous function defined on the compact set $\Omega$, then for any given small constant $\varepsilon>0$, there exists a fuzzy logic system such that $\sup _{x \in \Omega}\left|f(x)-\theta^{\mathrm{T}} \xi(x)\right| \leq \varepsilon$.

\section{Observer-Based Adaptive Control Scheme Design}

In order to estimate the unmeasured states, we design a linear state observer for $k t h$ as follows [10]:

$$
\left\{\begin{array}{l}
\dot{\hat{x}}_{k, 1}=\widehat{x}_{k, 2}-\ell_{k, 1}\left(\widehat{x}_{k, 1}-y_{k}\right), \\
\dot{\bar{x}}_{k, 2}=\widehat{x}_{k, 3}-\ell_{k, 2}\left(\widehat{x}_{k, 1}-y_{k}\right), \\
\vdots \\
\dot{\hat{x}}_{k, m_{k}-1}=\widehat{x}_{k, m_{k}}-\ell_{k, m_{k}-1}\left(\widehat{x}_{k, 1}-y_{k}\right), \\
\dot{\hat{x}}_{k, m_{k}}=D_{k} u_{k}-\ell_{k, m_{k}}\left(\hat{x}_{k, 1}-y_{k}\right),
\end{array}\right.
$$

where $\hat{x}_{k, l}\left(l=1,2, \ldots, m_{k}\right)$ is the estimation of $x_{k, l}$. $\ell_{k, 1}, \ldots, \ell_{k, m_{k}}$ are the observer design parameters. Suppose that $s$ th switched subsystem is active. Define observer error vector as $\underline{\tilde{x}}_{k}=\underline{x}_{k}-\underline{\hat{x}}_{k}$, where $\underline{x}_{k}=\left[x_{k, 1}, x_{k, 2}, \ldots, x_{k, m_{k}}\right]^{T}$, $\underline{\hat{x}}_{k}=\left[\hat{x}_{k, 1}, \widehat{x}_{k, 2}, \ldots, \hat{x}_{k, m_{k}}\right]^{T}$. 
Then, from (1) and (8), we can have

$$
\underline{\dot{\tilde{x}}}_{k}=A_{k} \underline{\tilde{x}}_{k}+F_{k}^{s}+H_{k}^{s} \text {, }
$$

where $A_{k}=\left[\begin{array}{ccc}-\ell_{k, 1} & & \\ \vdots & & I_{\left(m_{k}-1\right) \times\left(m_{k}-1\right)} \\ -\ell_{k, m_{k}} & 0 & \end{array}\right], \quad F_{k}^{s}=\left[f_{k, 1}^{s}\left(\underline{x}_{k}\right)\right.$, $\left.\ldots, f_{k, m_{k}}^{s}\left(\underline{x}_{k}\right)\right]^{T}, H_{k}^{s}=\left[h_{k, 1}^{s}(y), \ldots, h_{k, m_{k}}^{s}(y)\right]^{T}$. We can choose appropriate parameters $\bar{\ell}_{k, 1}, \ldots, \ell_{k, m_{k}}$ to ensure $A_{k}$ is a Hurwitz matrix, that is, for any given positive definite matrix $Q_{k}=Q_{k}^{T}>0$, there exists a positive definite matrix $P_{k}=P_{k}^{T}>0$, such that

$$
A_{k}^{T} P_{k}+P_{k} A_{k}=-Q_{k}
$$

Choose Lyapunov function candidate as [10]

$$
V_{0}=\sum_{k=1}^{M} \underline{\tilde{x}}_{k}^{T} P_{k} \underline{\tilde{x}}_{k}
$$

Then, we can obtain the time derivative of $V_{0}$ :

$$
\dot{V}_{0}=-\sum_{k=1}^{M} \underline{\tilde{x}}_{k}^{T} Q_{k} \underline{\tilde{x}}_{k}+\sum_{k=1}^{M} 2 \underline{\tilde{x}}_{k}^{T} P_{k}\left(F_{k}^{s}+H_{k}^{s}\right) \text {. }
$$

According to Young's inequality, we have

$$
2 \underline{\tilde{x}}_{k}^{T} P_{k} F_{k}^{s} \leq\left\|\underline{\tilde{x}}_{k}\right\|^{2}+\left\|P_{k}\right\|^{2} \sum_{j=1}^{m_{k}}\left(f_{k, j}^{s}\left(\underline{x}_{k}\right)\right)^{2} \text {. }
$$

Based on Assumption 2, $f_{k, j}^{s}\left(\underline{x}_{k}\right)$ satisfies

$$
f_{k, j}^{s}\left(\underline{x}_{k}\right)=y_{k} \bar{f}_{k, j}^{s}\left(\underline{x}_{k}\right)
$$

where $\bar{f}_{k, j}^{s}\left(\underline{x}_{k}\right)$ is the unknown nonlinear function which can be approximated by a fuzzy logic system.

From (13) and (14), we have

$$
\begin{aligned}
\left\|P_{k}\right\|^{2} \sum_{j=1}^{m_{k}}\left(f_{k, j}^{s}\left(\underline{x}_{k}\right)\right)^{2} & =\left\|P_{k}\right\|^{2} \sum_{j=1}^{m_{k}} y_{k}^{2}\left(\bar{f}_{k, j}^{s}\left(\underline{x}_{k}\right)\right)^{2} \\
& =y_{k}\left\|P_{k}\right\|^{2} \sum_{j=1}^{m_{k}} y_{k}\left(\bar{f}_{k, j}^{s}\left(\underline{x}_{k}\right)\right)^{2} .
\end{aligned}
$$

Based on Assumption 1, we obtain

$$
\begin{aligned}
\sum_{k=1}^{M} 2 \underline{\tilde{x}}_{k}^{T} P_{k} H_{k}^{s} & \leq \sum_{k=1}^{M} \frac{1}{\lambda}\left\|\underline{\tilde{x}}_{k}\right\|^{2}+\sum_{k=1}^{M} \lambda\left\|P_{k}\right\|^{2}\left\|H_{k}^{s}\right\|^{2} \\
& \leq \frac{1}{\lambda} \sum_{k=1}^{M}\left\|\underline{\tilde{x}}_{k}\right\|^{2}+\bar{\lambda} \sum_{k=1}^{M} \sum_{l=1}^{m_{k}} \sum_{i=1}^{M}\left(y_{i} \hbar_{k, l, i}^{s}\left(y_{k}\right)\right)^{2} \\
& \leq \frac{1}{\lambda} \sum_{k=1}^{M}\left\|\underline{\tilde{x}}_{k}\right\|^{2}+\bar{\lambda} \sum_{i=1}^{M} \sum_{l=1}^{m_{k}} \sum_{k=1}^{M}\left(y_{i} \hbar_{i, l, k}^{s}\left(y_{k}\right)\right)^{2} \\
& \leq \frac{1}{\lambda} \sum_{k=1}^{M}\left\|\underline{\tilde{x}}_{k}\right\|^{2}+\bar{\lambda} \sum_{k=1}^{M} \sum_{i=1}^{M} \sum_{l=1}^{m_{k}} y_{k}^{2}\left(\hbar_{i, l, k}^{s}\left(y_{k}\right)\right)^{2} \\
& \leq \frac{1}{\lambda} \sum_{k=1}^{M}\left\|\underline{\tilde{x}}_{k}\right\|^{2}+\sum_{k=1}^{M} y_{k}^{2} \sum_{i=1}^{M} \sum_{l=1}^{m_{k}} \bar{\lambda}\left(\hbar_{i, l, k}^{s}\left(y_{k}\right)\right)^{2},
\end{aligned}
$$

where $\lambda>0$ is the design parameter and $\bar{\lambda}=\lambda \max _{1 \leq k \leq M}$ $\left(\left\|P_{k}\right\|\right)^{2}$

Substituting equations (13)-(16) into (12) results in

$$
\begin{aligned}
\dot{V}_{0} & \leq \sum_{k=1}^{M}\left\{-\underline{\tilde{x}}_{k}^{T} Q_{k} \underline{\tilde{x}}_{k}+\left(1+\frac{1}{\lambda}\right)\left\|\underline{\tilde{x}}_{k}\right\|^{2}+y_{k}^{2} \sum_{i=1}^{M} \sum_{l=1}^{m_{k}} \bar{\lambda}\left(\hbar_{i, l, k}^{s}\left(y_{k}\right)\right)^{2}+y_{k}\left\|P_{k}\right\|^{2} \sum_{j=1}^{m_{k}} y_{k}\left(\bar{f}_{k, j}^{s}\left(\underline{x}_{k}\right)\right)^{2}\right\} \\
& \leq \sum_{k=1}^{M}\left\{-\underline{\tilde{x}}_{k}^{T} Q_{k} \underline{\tilde{x}}_{k}+\left(1+\frac{1}{\lambda}\right)\left\|\underline{\tilde{x}}_{k}\right\|^{2}+y_{k}\left[y_{k} \sum_{i=1}^{M} \sum_{l=1}^{m_{k}} \bar{\lambda}\left(\hbar_{i, l, k}^{s}\left(y_{k}\right)\right)^{2}+\left\|P_{k}\right\|^{2} \sum_{j=1}^{m_{k}} y_{k}\left(\bar{f}_{k, j}^{s}\left(\underline{x}_{k}\right)\right)^{2}+\sum_{i=1}^{M} y_{k}\left(\hbar_{i, 1, k}^{s}\left(y_{k}\right)\right)^{2}\right]-\sum_{i=1}^{M} y_{k}^{2}\left(\hbar_{i, 1, k}^{s}\left(y_{k}\right)\right)^{2}\right\} .
\end{aligned}
$$

Let $\quad \Phi_{k, s}\left(\underline{x}_{k}\right)=y_{k} \sum_{i=1}^{M} \sum_{l=1}^{m_{k}} \bar{\lambda} \quad\left(\hbar_{i, l, k}^{s}\left(y_{k}\right)\right)^{2}+\left\|P_{k}\right\|^{2}$ $\sum_{j=1}^{m_{k}} y_{k}\left(\bar{f}_{k, j}^{s}\left(\underline{x}_{k}\right)\right)^{2}+\sum_{i=1}^{M} y_{k}\left(\hbar_{i, 1, k}^{s}\left(y_{k}\right)\right)^{2}$. By using fuzzy system $\widehat{f}_{k, s}\left(\underline{x}_{k} \mid \theta_{k, s}\right)=\theta_{k, s}^{T} \xi_{k, s}\left(\underline{x}_{k}\right)$ to approximate $\Phi_{k, s}\left(\underline{x}_{k}\right)$, there exists optimal parameter vector

$$
\theta_{k, s}^{*}=\arg \min _{\theta_{k, s} \in \Omega_{k, s}}\left[\sup _{\underline{x}_{k} \in U_{k}}\left|\widehat{f}_{k, s}\left(\underline{x}_{k} \mid \theta_{k, s}\right)-\Phi_{k, s}\left(\underline{x}_{k}\right)\right|\right],
$$

where $\Omega_{k, s}$ and $U_{k}$ are the compact sets of $\theta_{k, s}$ and $\underline{x}_{k}$, respectively. Then, the minimal error can be written as

$$
\varepsilon_{k, s}\left(\underline{x}_{k}\right)=\Phi_{k, s}\left(\underline{x}_{k}\right)-\widehat{f}_{k, s}\left(\underline{x}_{k} \mid \theta_{k, s}^{*}\right),
$$

where $\left|\varepsilon_{k, s}\left(\underline{x}_{k}\right)\right| \leq \varepsilon_{k, s}^{*}$ and $\varepsilon_{k, s}^{*}$ is the unknown positive constant.

From (18) and (19), we can obtain

$$
\dot{V}_{0} \leq \sum_{k=1}^{M}\left\{-\left(\lambda_{\min }\left(Q_{k}\right)-1-\frac{1}{\lambda}\right)\left\|\underline{\tilde{x}}_{k}\right\|^{2}+y_{k}\left[\theta_{k, s}^{* T} \xi_{k, s}\left(\underline{x}_{k}\right)+\varepsilon_{k, s}\left(\underline{x}_{k}\right)\right]-\sum_{i=1}^{M} y_{k}^{2}\left(\hbar_{i, 1, k}^{s}\left(y_{k}\right)\right)^{2}\right\}
$$


where $\lambda_{\min }\left(Q_{k}\right)$ is the minimal eigenvalue of matrix $Q_{k}$.

\section{Adaptive Control Law Design}

Define the tracking error $z_{k, 1}$, virtual error $z_{k, l}$, virtual control law $\alpha_{k, l-1}$, and first-order filters as

$$
\left\{\begin{array}{l}
z_{k, 1}=y_{k}-y_{k, d}, \\
z_{k, l}=\hat{x}_{k, l}-\omega_{k, l-1}, \\
v_{k, l-1} \dot{\omega}_{k, l-1}+\omega_{k, l-1}=\alpha_{k, l-1}, \\
\omega_{k, l-1}(0)=\alpha_{k, l-1}(0),
\end{array}\right.
$$

where $l=2, \ldots, m_{k} \cdot v_{k, l-1}$ is the time constant of the filter, that is, by letting $\alpha_{k, l-1}$ pass through a filter that has the time constant $v_{k, l-1}$, we can obtain $\omega_{k, l-1}$.

Step 1. Define $\omega_{k, 0}=y_{k, d}$. From (21), we have

$$
\begin{aligned}
v_{k, 1} \dot{\omega}_{k, 1}+\omega_{k, 1} & =\alpha_{k, 1}, \\
\omega_{k, 1}(0) & =\alpha_{k, 1}(0) .
\end{aligned}
$$

Define $e_{k, 1}$ as the first filter output error; then, we can obtain $e_{k, 1}=\omega_{k, 1}-\alpha_{k, 1}$ and $\dot{\omega}_{k, 1}=-\left(e_{k, 1} / v_{k, 1}\right)$.

The time derivative of $z_{k, 1}$ is as follows:

$$
\begin{aligned}
\dot{z}_{k, 1} & =x_{k, 2}+f_{k, 1}^{s}+h_{k, 1}^{s}-\dot{\omega}_{k, 0} \\
& =z_{k, 2}+e_{k, 1}+\alpha_{k, 1}+\tilde{x}_{k, 2}+f_{k, 1}^{s}\left(\underline{x}_{k}\right)+h_{k, 1}^{s}(\underline{y})-\dot{y}_{k, d} .
\end{aligned}
$$

By using fuzzy system $\widehat{F}_{k, s}\left(\underline{x}_{k} \mid \varphi_{k, s}\right)=\varphi_{k, s}^{T} \zeta_{k, s}\left(\underline{x}_{k}\right)$ to approximate $f_{k, 1}^{s}\left(\underline{x}_{k}\right)$ and defining $\varphi_{k, s}^{*}$ as the optimal parameter vector, we have

$$
\varphi_{k, s}^{*}=\arg \min _{\varphi_{k, s} \in \bar{\Omega}_{k, s}}\left[\sup _{x_{k} \in U_{k}}\left|\widehat{F}_{k, s}\left(\underline{x}_{k} \mid \varphi_{k, s}\right)-f_{k, 1}^{s}\left(\underline{x}_{k}\right)\right|\right],
$$

where $\bar{\Omega}_{k, s}$ is the compact set of $\varphi_{k, s}$. Then, the minimal approximation error is as follows:

$$
\delta_{k, s}\left(\underline{x}_{k}\right)=f_{k, 1}^{s}\left(\underline{x}_{k}\right)-\widehat{F}_{k, s}\left(\underline{x}_{k} \mid \varphi_{k, s}^{*}\right),
$$

where $\left|\delta_{k, s}\left(\underline{x}_{k}\right)\right| \leq \delta_{k, s}^{*}$ and $\delta_{k, s}^{*}$ is the unknown positive constant.

Choose Lyapunov function candidate as

$$
V_{1}=V_{0}+\sum_{k=1}^{M}\left[\frac{1}{2} z_{k, 1}^{2}+\frac{1}{2} e_{k, 1}^{2}+\frac{1}{2 \gamma_{k, 1}} \widetilde{\widetilde{\omega}}_{k, 1}^{2}+\frac{1}{2 \gamma_{k, 2}} \widetilde{\widetilde{\omega}}_{k, 2}^{2}\right]
$$

where $\gamma_{k, 1}>0$ and $\gamma_{k, 2}>0$ are design parameters, $\varpi_{k, 1}^{*}=\max _{s \in N}\left\{\theta_{k, s}^{* T} \theta_{k, s}^{*}\right\}, \omega_{k, 2}^{*}=\max _{s \in N}\left\{\varphi_{k, s}^{* T} \varphi_{k, s}^{*}\right\}$, and $\varpi_{k, 1}$ and $\Phi_{k, 2}$ are estimations of $\omega_{k, 1}^{*}$ and $\omega_{k, 2}^{*}$, respectively. Define $\widetilde{\varpi}_{k, 1}=\varpi_{k, 1}^{*}-\varpi_{k, 1}, \widetilde{\omega}_{k, 2}=\varpi_{k, 2}^{*}-\varpi_{k, 2}$.

Now, we can infer that $\dot{V}_{1}$ satisfies

$$
\begin{aligned}
& \dot{V}_{1}=\dot{V}_{0}+\sum_{k=1}^{M}\left[z_{k, 1} \dot{z}_{k, 1}+e_{k, 1} \dot{e}_{k, 1}-\frac{1}{\gamma_{k, 1}} \widetilde{\mathrm{\omega}}_{k, 1} \dot{\mathrm{\omega}}_{k, 1}-\frac{1}{\gamma_{k, 2}} \widetilde{\mathrm{\omega}}_{k, 2} \dot{\mathrm{\omega}}_{k, 2}\right] \\
& =\dot{V}_{0}+\sum_{k=1}^{M}\left[z_{k, 1}\left(z_{k, 2}+e_{k, 1}+\alpha_{k, 1}+\tilde{x}_{k, 2}+f_{k, 1}^{s}\left(\underline{x}_{k}\right)+h_{k, 1}^{s}(\underline{y})-\dot{y}_{k, d}\right)+e_{k, 1} \dot{e}_{k, 1}-\frac{1}{\gamma_{k, 1}} \widetilde{\omega}_{k, 1} \dot{\grave{\omega}}_{k, 1}-\frac{1}{\gamma_{k, 2}} \widetilde{\omega}_{k, 2} \dot{\Phi}_{k, 2}\right] \text {. }
\end{aligned}
$$
have

According to Young's inequality and Assumption 1, we

$$
\sum_{k=1}^{M} z_{k, 1} h_{k, 1}^{s}(\underline{y}) \leq \sum_{k=1}^{M} \frac{z_{k, 1}^{2}}{4}+\sum_{k=1}^{M}\left(h_{k, 1}^{s}(\underline{y})\right)^{2} \leq \sum_{k=1}^{M} \frac{z_{k, 1}^{2}}{4}+\sum_{k=1}^{M} \sum_{i=1}^{M} y_{k}^{2}\left(\hbar_{k, 1, i}^{s}\left(y_{k}\right)\right)^{2} .
$$

Substituting (28) into (27), we obtain

$$
\begin{aligned}
& \dot{V}_{1}=\dot{V}_{0}+\sum_{k=1}^{M}\left[z_{k, 1} \dot{z}_{k, 1}+e_{k, 1} \dot{e}_{k, 1}-\frac{1}{\gamma_{k, 1}} \widetilde{\varpi}_{k, 1} \dot{\omega}_{k, 1}-\frac{1}{\gamma_{k, 2}} \widetilde{\varpi}_{k, 2} \dot{\omega}_{k, 2}\right] \leq \sum_{k=1}^{M}\left\{-\left(\lambda_{\min }\left(Q_{k}\right)-1-\frac{1}{\lambda}\right)\left\|\underline{\underline{x}}_{k}\right\|^{2}+y_{k}\left[\theta_{k, s}^{T *} \xi_{k, s}\left(\underline{x}_{k}\right)+\varepsilon_{k, s}\left(\underline{x}_{k}\right)\right]+\frac{z_{k, 1}^{2}}{4}\right\} \\
& +\sum_{k=1}^{M}\left[z_{k, 1}\left(z_{k, 2}+e_{k, 1}+\alpha_{k, 1}+\widetilde{x}_{k, 2}+\varphi_{k, s}^{* T} \xi_{k, s}\left(\underline{x}_{k}\right)+\delta_{k, s}\left(\underline{x}_{k}\right)-\dot{y}_{k, d}\right)+e_{k, 1} \dot{e}_{k, 1}-\frac{1}{\gamma_{k, 1}} \widetilde{\omega}_{k, 1} \dot{\omega}_{k, 1}-\frac{1}{\gamma_{k, 2}} \widetilde{\omega}_{k, 2} \dot{\mathrm{\omega}}_{k, 2}\right] .
\end{aligned}
$$


Based on $\xi_{k, s}^{T}(\cdot) \xi_{k, s}(\cdot) \leq 1$ and Young's inequality, we can infer the following inequalities:

$$
\begin{aligned}
y_{k}\left[\theta_{k, s}^{* T} \xi_{k, s}\left(\underline{x}_{k}\right)+\varepsilon_{k, s}\left(\underline{x}_{k}\right)\right] & \leq z_{k, 1}\left[\theta_{k, s}^{* T} \xi_{k, s}\left(\underline{x}_{k}\right)+\varepsilon_{k, s}\left(\underline{x}_{k}\right)\right]+y_{k, d}\left[\theta_{k, s}^{* T} \xi_{k, s}\left(\underline{x}_{k}\right)+\varepsilon_{k, s}\left(\underline{x}_{k}\right)\right] \\
& \leq \frac{z_{k, 1}^{2} \Phi_{k, 1}^{*}}{4 \tau_{k}}+\frac{z_{k, 1}^{2}}{4}+\frac{y_{k, d}^{2}}{2}+\Phi_{k, 1}^{*}+2 \varepsilon_{k}^{* 2}+\tau_{k}, \\
z_{k, 1}\left[\varphi_{k, s}^{* T} \xi_{k, s}\left(\underline{x}_{k}\right)+\delta_{k, s}\left(\underline{x}_{k}\right)\right] & \leq \frac{z_{k, 1}^{2} \Phi_{k, 2}^{*}}{4 \tau_{k}}+\frac{z_{k, 1}^{2}}{4}+\delta_{k}^{* 2}+\tau_{k}, \\
z_{k, 1} \tilde{x}_{k, 2} \leq & \frac{1}{\lambda_{k}}\left\|\underline{\tilde{x}}_{k}\right\|^{2}+\lambda_{k} \frac{z_{k, 1}^{2}}{4},
\end{aligned}
$$
where $\varepsilon_{k}^{*}=\max _{s \in N}\left\{\varepsilon_{k, s}^{* 2}\right\}, \delta_{k}^{*}=\max _{s \in N}\left\{\delta_{k, s}^{* 2}\right\}$, and $\tau_{k}>0$ and $\lambda_{k}>0$
are design parameters.

Substituting (30)-(32) into (29) results in

$$
\begin{aligned}
\dot{V}_{1} \leq & \sum_{k=1}^{M}\left\{-\left(\lambda_{\min }\left(Q_{k}\right)-1-\frac{1}{\lambda}-\frac{1}{\lambda_{k}}\right)\left\|\tilde{x}_{k}\right\|^{2}\right\} \\
& +\sum_{k=1}^{M}\left[z_{k, 1}\left(z_{k, 2}+e_{k, 1}+\alpha_{k, 1}+\frac{z_{k, 1} \omega_{k, 1}^{*}}{4 \tau_{k}}+\frac{z_{k, 1} \omega_{k, 2}^{*}}{4 \tau_{k}}+\frac{\left(3+\lambda_{k}\right) z_{k, 1}}{4}-\dot{y}_{k, d}\right)+e_{k, 1} \dot{e}_{k, 1}\right. \\
& \left.-\frac{1}{\gamma_{k, 1}} \widetilde{\omega}_{k, 1} \dot{\omega}_{k, 1}-\frac{1}{\gamma_{k, 2}} \widetilde{\varpi}_{k, 2} \dot{\mathrm{\omega}}_{k, 2} \frac{\bar{y}_{k, d}^{2}}{2}+\omega_{k, 1}^{*}+2 \varepsilon_{k}^{* 2}+\delta_{k}^{* 2}+2 \tau_{k}\right],
\end{aligned}
$$

where $\bar{y}_{k, d}=\max _{t \in[0,+\infty]}\left\{y_{k, d}(t)\right\}$ is a constant. Then, (33) can be rearranged as

$$
\begin{aligned}
\dot{V}_{1} \leq & \sum_{k=1}^{M}\left\{-q_{k, 1}\left\|\underline{\tilde{x}}_{k}\right\|^{2}\right\}+\sum_{k=1}^{M}\left[z_{k, 1}\left(z_{k, 2}+e_{k, 1}+\alpha_{k, 1}+\frac{z_{k, 1} \oplus_{k, 1}}{4 \tau_{k}}+\frac{z_{k, 1} \oplus_{k, 2}}{4 \tau_{k}}+\frac{\left(3+\lambda_{k}\right) z_{k, 1}}{4}-\dot{y}_{k, d}\right)\right. \\
& \left.+\frac{1}{\gamma_{k, 1}} \widetilde{\omega}_{k, 1}\left(\frac{\gamma_{k, 1} z_{k, 1}^{2}}{4 \tau_{k}}-\dot{\omega}_{k, 1}\right)+\frac{1}{\gamma_{k, 2}} \widetilde{\omega}_{k, 2}\left(\frac{\gamma_{k, 2} z_{k, 1}^{2}}{4 \tau_{k}}-\dot{\omega}_{k, 2}\right)+e_{k, 1} \dot{e}_{k, 1}+d_{k, 1}\right],
\end{aligned}
$$

where $q_{k, 1}=\lambda_{\min }\left(Q_{k}\right)-1-(1 / \lambda)-\left(1 / \lambda_{k}\right)$ and $d_{k, 1}=\left(\bar{y}_{k, d}^{2} /\right.$ 2) $+\omega_{k, 1}^{*}+2 \varepsilon_{k}^{* 2}+\delta_{k}^{* 2}+2 \tau_{k}$.

$$
\begin{aligned}
& \dot{\oplus}_{k, 1}=\frac{\gamma_{k, 1} z_{k, 1}^{2}}{4 \tau_{k}}-2 \sigma_{k, 1} \oplus_{k, 1}, \\
& \dot{\varpi}_{k, 2}=\frac{\gamma_{k, 2} z_{k, 1}^{2}}{4 \tau_{k}}-2 \sigma_{k, 2} \oplus_{k, 2},
\end{aligned}
$$

$$
-\frac{\left(3+\lambda_{k}\right) z_{k, 1}}{4}+\dot{y}_{k, d} \text {, }
$$

where $c_{k, 1}>0, \sigma_{k, 1}>0$, and $\sigma_{k, 2}>0$ are design parameters. Substituting (35)-(37) into (34) results in 


$$
\begin{aligned}
\dot{V}_{1} & \leq \sum_{k=1}^{M}\left\{-q_{k, 1}\left\|\underline{\tilde{x}}_{k}\right\|^{2}\right\}+\sum_{k=1}^{M}\left[-c_{k, 1} z_{k, 1}^{2}+z_{k, 1} z_{k, 2}+z_{k, 1} e_{k, 1}+\frac{2 \sigma_{k, 1}}{\gamma_{k, 1}} \widetilde{\omega}_{k, 1} \varpi_{k, 1}+\frac{2 \sigma_{k, 2}}{\gamma_{k, 2}} \widetilde{\varpi}_{k, 2} \oplus_{k, 2}+e_{k, 1}\left(-\frac{e_{k, 1}}{v_{k, 1}}-\dot{\alpha}_{k, 1}\right)+d_{k, 1}\right] \\
& \leq \sum_{k=1}^{M}\left\{-q_{k, 1}\left\|\underline{\tilde{x}}_{k}\right\|^{2}\right\}+\sum_{k=1}^{M}\left[-c_{k, 1} z_{k, 1}^{2}+z_{k, 1} z_{k, 2}+z_{k, 1} e_{k, 1}+\frac{2 \sigma_{k, 1}}{\gamma_{k, 1}} \widetilde{\omega}_{k, 1} \varpi_{k, 1}+\frac{2 \sigma_{k, 2}}{\gamma_{k, 2}} \widetilde{\omega}_{k, 2} \oplus_{k, 2}-\frac{e_{k, 1}^{2}}{v_{k, 1}}+e_{k, 1}^{2}+\frac{1}{4} \psi_{k, 1}^{2}+d_{k, 1}\right],
\end{aligned}
$$

where $\psi_{k, 1}$ is the maximum absolute value of $\dot{\alpha}_{k, 1}$.

According to Young's inequality,

$$
\begin{aligned}
& \frac{2 \sigma_{k, 1}}{\gamma_{k, 1}} \widetilde{\varpi}_{k, 1} \oplus_{k, 1} \leq-\frac{\sigma_{k, 1}}{\gamma_{k, 1}} \oplus_{k, 1}^{2}+\frac{\sigma_{k, 1}}{\gamma_{k, 1}} \oplus_{k, 1}^{* 2}, \\
& \frac{2 \sigma_{k, 2}}{\gamma_{k, 2}} \widetilde{\omega}_{k, 2} \oplus_{k, 2} \leq-\frac{\sigma_{k, 2}}{\gamma_{k, 2}} \varpi_{k, 2}^{2}+\frac{\sigma_{k, 2}}{\gamma_{k, 2}} \omega_{k, 2}^{* 2}, \\
& z_{k, 1} e_{k, 1} \leq z_{k, 1}^{2}+\frac{1}{4} e_{k, 1}^{2} .
\end{aligned}
$$

Substituting (39)-(41) into (38) results in

$$
\begin{aligned}
\dot{V}_{1} \leq & \sum_{k=1}^{M}\left\{-q_{k, 1}\left\|\underline{\tilde{x}}_{k}\right\|^{2}\right\} \\
& +\sum_{k=1}^{M}\left[-\left(c_{k, 1}-1\right) z_{k, 1}^{2}+z_{k, 1} z_{k, 2}+\left(\frac{5}{4}-\frac{1}{v_{k, 1}}\right) e_{k, 1}^{2}\right. \\
& \left.-\sum_{i=1}^{2} \frac{\sigma_{k, i}}{\gamma_{k, i}} \Phi_{k, i}^{2}+\sum_{i=1}^{2} \frac{\sigma_{k, i}}{\gamma_{k, i}} \Phi_{k, i}^{* 2}+\frac{1}{4} \psi_{k, 1}^{2}+d_{k, 1}\right] .
\end{aligned}
$$

Substituting the inequality

$$
-\frac{\sigma_{k, i}}{2 \gamma_{k, i}} \widetilde{\varpi}_{k, i}^{2} \geq-\frac{\sigma_{k, i}}{\gamma_{k, i}} \oplus_{k, i}^{2}-\frac{\sigma_{k, i}}{\gamma_{k, i}} \oplus_{k, i}^{* 2}
$$

into equation (42) results in

$$
\begin{aligned}
\dot{V}_{1} \leq & \sum_{k=1}^{M}\left\{-q_{k, 1}\left\|\underline{\tilde{x}}_{k}\right\|^{2}\right\} \\
& +\sum_{k=1}^{M}\left[-\left(c_{k, 1}-1\right) z_{k, 1}^{2}+z_{k, 1} z_{k, 2}+\left(\frac{5}{4}-\frac{1}{v_{k, 1}}\right) e_{k, 1}^{2}\right. \\
& \left.-\sum_{i=1}^{2} \frac{\sigma_{k, i}}{2 \gamma_{k, i}} \widetilde{\omega}_{k, i}^{2}+\sum_{i=1}^{2} \frac{2 \sigma_{k, i}}{\gamma_{k, i}} \varpi_{k, i}^{* 2}+\frac{1}{4} \psi_{k, 1}^{2}+d_{k, 1}\right] .
\end{aligned}
$$

Step 2. Define $z_{k, 2}=\widehat{x}_{k, 2}-\omega_{k, 1}$ and $z_{k, 3}=\widehat{x}_{k, 3}-\omega_{k, 2}$. From (21), we have

$$
\begin{aligned}
v_{k, 2} \dot{\omega}_{k, 2}+\omega_{k, 2} & =\alpha_{k, 2}, \\
\omega_{k, 2}(0) & =\alpha_{k, 2}(0) .
\end{aligned}
$$

Define $e_{k, 2}$ as the filter output error; then, we have $e_{k, 2}=$ $\omega_{k, 2}-\alpha_{k, 2}$ and $\dot{\omega}_{k, 2}=-\left(e_{k, 2} / v_{k, 2}\right)$.

The time derivative of $z_{k, 2}$ is as follows:

$$
\begin{aligned}
\dot{z}_{k, 2} & =\widehat{x}_{k, 3}-\ell_{k, 2}\left(\hat{x}_{k, 1}-y_{k}\right)-\dot{\omega}_{k, 1} \\
& =z_{k, 3}+\alpha_{k, 2}+e_{k, 2}-\ell_{k, 2}\left(\widehat{x}_{k, 1}-y_{k}\right)-\dot{\omega}_{k, 1} .
\end{aligned}
$$

Choose Lyapunov function candidate as

$$
V_{2}=V_{1}+\sum_{k=1}^{M}\left[\frac{1}{2} z_{k, 2}^{2}+\frac{1}{2} e_{k, 2}^{2}\right] .
$$

The time derivative of $V_{2}$ is as follows:

$$
\begin{aligned}
\dot{V}_{2}= & \dot{V}_{1}+\sum_{k=1}^{M}\left(z_{k, 2} \dot{z}_{k, 2}+e_{k, 2} \dot{e}_{k, 2}\right) \\
= & \dot{V}_{1}+\sum_{k=1}^{M}\left[z_{k, 2}\left(z_{k, 3}+\alpha_{k, 2}+e_{k, 2}-\ell_{k, 2}\left(\hat{x}_{k, 1}-y_{k}\right)-\dot{\omega}_{k, 1}\right)+e_{k, 2} \dot{e}_{k, 2}\right] \leq \dot{V}_{1} \\
& +\sum_{k=1}^{M}\left[z_{k, 2}\left(z_{k, 3}+\alpha_{k, 2}+e_{k, 2}-\ell_{k, 2}\left(\hat{x}_{k, 1}-y_{k}\right)-\dot{\omega}_{k, 1}\right)-\frac{e_{k, 2}^{2}}{v_{k, 2}}+e_{k, 2}^{2}+\frac{1}{4} \psi_{k, 2}^{2}\right],
\end{aligned}
$$

where $\psi_{k, 2}$ is the maximum absolute value of $\dot{\alpha}_{k, 2}$.

According to Young's inequality, we have 


$$
\begin{aligned}
\dot{V}_{2} \leq & \dot{V}_{1}+\sum_{k=1}^{M}\left[z_{k, 2}\left(z_{k, 3}+\alpha_{k, 2}-\ell_{k, 2}\left(\widehat{x}_{k, 1}-y_{k}\right)-\dot{\omega}_{k, 1}\right)+z_{k, 2}^{2}+\frac{1}{4} e_{k, 2}^{2}-\frac{e_{k, 2}^{2}}{v_{k, 2}}+e_{k, 2}^{2}+\frac{1}{4} \psi_{k, 2}^{2}\right] \leq \sum_{k=1}^{M}\left\{-q_{k, 1}\left\|\underline{\tilde{x}}_{k}\right\|^{2}\right\} \\
& +\sum_{k=1}^{M}\left[-\left(c_{k, 1}-1\right) z_{k, 1}^{2}+z_{k, 1} z_{k, 2}+\left(\frac{5}{4}-\frac{1}{v_{k, 1}}\right) e_{k, 1}^{2}-\sum_{i=1}^{2} \frac{\sigma_{k, i}}{2 \gamma_{k, i}} \widetilde{\omega}_{k, i}^{2}+\sum_{i=1}^{2} \frac{2 \sigma_{k, i}}{\gamma_{k, i}} \omega_{k, i}^{* 2}+\frac{1}{4} \psi_{k, 2}^{2}+d_{k, 1}\right] \\
& +\sum_{k=1}^{M}\left[z_{k, 2}\left(z_{k, 3}+\alpha_{k, 2}-\ell_{k, 2}\left(\hat{x}_{k, 1}-y_{k}\right)-\dot{\omega}_{k, 1}\right)+z_{k, 2}^{2}+\left(\frac{5}{4}-\frac{1}{v_{k, 2}}\right) e_{k, 2}^{2}+\frac{1}{4} \psi_{k, 2}^{2}\right] .
\end{aligned}
$$

Design $\alpha_{k, 2}$ as follows:

$$
\alpha_{k, 2}=-c_{k, 2} z_{k, 2}-z_{k, 1}+\ell_{k, 2}\left(\widehat{x}_{k, 1}-y_{k}\right)-\frac{\omega_{k, 1}-\alpha_{k, 1}}{v_{k, 1}},
$$

where $c_{k, 2}>0$ is the design parameter.

Substituting (50) into (49) leads to

$$
\begin{aligned}
\dot{V}_{2} \leq & \sum_{k=1}^{M}\left\{-q_{k, 1}\left\|\underline{\tilde{x}}_{k}\right\|^{2}\right\}+\sum_{k=1}^{M}\left[-\left(c_{k, 1}-1\right) z_{k, 1}^{2}+z_{k, 1} z_{k, 2}+\left(\frac{5}{4}-\frac{1}{v_{k, 1}}\right) e_{k, 1}^{2}-\sum_{i=1}^{2} \frac{\sigma_{k, i}}{2 \gamma_{k, i}} \widetilde{\omega}_{k, i}^{2}+\sum_{i=1}^{2} \frac{2 \sigma_{k, i}}{\gamma_{k, i}} \oplus_{k, i}^{* 2}+\frac{1}{4} \psi_{k, 1}^{2}+d_{k, 1}\right] \\
& +\sum_{k=1}^{M}\left[z_{k, 2} z_{k, 3}-z_{k, 2} z_{k, 1}-\left(c_{k, 2}-1\right) z_{k, 2}^{2}+\left(\frac{5}{4}-\frac{1}{v_{k, 2}}\right) e_{k, 2}^{2}+\frac{1}{4} \psi_{k, 2}^{2}\right] \leq \sum_{k=1}^{M}\left\{-q_{k, 1}\left\|\underline{\widetilde{x}}_{k}\right\|^{2}\right\} \\
& +\sum_{k=1}^{M}\left[-\sum_{i=1}^{2}\left(c_{k, i}-1\right) z_{k, i}^{2}+z_{k, 2} z_{k, 3}+\sum_{i=1}^{2}\left(\frac{5}{4}-\frac{1}{v_{k, i}}\right) e_{k, i}^{2}-\sum_{i=1}^{2} \frac{\sigma_{k, i}}{2 \gamma_{k, i}} \widetilde{\omega}_{k, i}^{2}+\sum_{i=1}^{2} \frac{2 \sigma_{k, i}}{\gamma_{k, i}} \oplus_{k, i}^{* 2}+\sum_{i=1}^{2} \frac{1}{4} \psi_{k, i}^{2}+d_{k, 1}\right] .
\end{aligned}
$$

Step $l$ th $\left(l=3, \ldots, m_{k}-1\right)$ : define $z_{k, l}=\widehat{x}_{k, l}-\omega_{k, l-1}$ and $z_{k, l+1}=\widehat{x}_{k, l+1}-\omega_{k, l}$. From (21), we have

$$
\begin{aligned}
v_{k, l} \dot{\omega}_{k, l}+\omega_{k, l} & =\alpha_{k, l}, \\
\omega_{k, l}(0) & =\alpha_{k, l}(0) .
\end{aligned}
$$

Define the filter output error as $e_{k, l}$; then, we obtain $e_{k, l}=$ $\omega_{k, l}-\alpha_{k, l}$ and $\dot{\omega}_{k, l}=-\left(e_{k, l} / v_{k, l}\right)$.

The time derivative of $z_{k, l}$ is as follows:

$$
\begin{aligned}
\dot{z}_{k, l} & =\widehat{x}_{k, l+1}-\ell_{k, l}\left(\widehat{x}_{k, 1}-y_{k}\right)-\dot{\omega}_{k, l-1} \\
& =z_{k, l+1}+\alpha_{k, l}+e_{k, l}-\ell_{k, l}\left(\widehat{x}_{k, 1}-y_{k}\right)-\dot{\omega}_{k, l-1} .
\end{aligned}
$$

Choose Lyapunov function candidate as

$$
V_{l}=V_{l-1}+\sum_{k=1}^{M}\left[\frac{1}{2} z_{k, l}^{2}+\frac{1}{2} e_{k, l}^{2}\right] .
$$

The time derivative of $V_{l}$ is as follows:

$$
\begin{aligned}
\dot{V}_{l}= & \dot{V}_{l-1}+\sum_{k=1}^{M}\left(z_{k, l} \dot{z}_{k, l}+e_{k, l} \dot{e}_{k, l}\right) \\
= & \dot{V}_{l-1}+\sum_{k=1}^{M}\left[z _ { k , l } \left(z_{k, l+1}+\alpha_{k, l}+e_{k, l}\right.\right. \\
& \left.\left.-\ell_{k, l}\left(\hat{x}_{k, 1}-y_{k}\right)-\dot{\omega}_{k, l-1}\right)+e_{k, l} \dot{e}_{k, l}\right] .
\end{aligned}
$$

Design $\alpha_{k, l}$ as follows:

$$
\alpha_{k, l}=-c_{k, l} z_{k, l}-z_{k, l-1}+\ell_{k, l}\left(\widehat{x}_{k, 1}-y_{k}\right)-\frac{\omega_{k, l-1}-\alpha_{k, l-1}}{v_{k, l-1}},
$$

where $c_{k, l}>0$ is the design parameter.

Using the same recursive method in Step 2, we can get

$$
\dot{V}_{l} \leq \sum_{k=1}^{M}\left\{-q_{k, 1}\left\|\underline{\widetilde{x}}_{k}\right\|^{2}\right\}+\sum_{k=1}^{M}\left[-\sum_{i=1}^{l}\left(c_{k, i}-1\right) z_{k, i}^{2}+z_{k, l} z_{k, l+1}+\sum_{i=1}^{l}\left(\frac{5}{4}-\frac{1}{v_{k, i}}\right) e_{k, i}^{2}-\sum_{i=1}^{2} \frac{\sigma_{k, i}}{2 \gamma_{k, i}} \widetilde{\omega}_{k, i}^{2}+\sum_{i=1}^{2} \frac{2 \sigma_{k, i}}{\gamma_{k, i}} \omega_{k, i}^{* 2}+\sum_{i=1}^{l} \frac{1}{4} \psi_{k, i}^{2}+d_{k, 1}\right] .
$$

Step $m_{k} t h$ : define $z_{k, m_{k}}=\widehat{x}_{k, m_{k}}-\omega_{k, m_{k}-1}$, and we can get the time derivative of $z_{k, m_{k}}$ as follows: 


$$
\begin{aligned}
\dot{z}_{k, m_{k}} & =D_{k} u_{k}-\ell_{k, m_{k}}\left(\widehat{x}_{k, 1}-y_{k}\right)-\dot{\omega}_{k, m_{k}-1} \\
& =u_{k, d}+\left(\widetilde{d}_{k, m r}-\frac{u_{k, d}+\widehat{d}_{k, m r}}{\widehat{m}_{k, r}} \widetilde{m}_{k, r}\right) \delta_{k}-\left(\widetilde{d}_{k, m l}+\frac{u_{k, d}-\widehat{d}_{k, m l}}{\widehat{m}_{k, l}} \widetilde{m}_{k, l}\right)\left(1-\delta_{k}\right)+\varepsilon_{k, d}-\ell_{k, m_{k}}\left(\widehat{x}_{k, 1}-y_{k}\right)-\dot{\omega}_{k, m_{k}-1} .
\end{aligned}
$$

Choose Lyapunov function candidate as

$$
\begin{aligned}
V_{m_{k}}= & V_{m_{k}-1}+\sum_{k=1}^{M}\left[\frac{1}{2} z_{k, m_{k}}^{2}+\frac{1}{2 \eta_{k, 1}} \tilde{m}_{k, r}^{2}\right. \\
& \left.+\frac{1}{2 \eta_{k, 2}} \tilde{m}_{k, l}^{2}+\frac{1}{2 \eta_{k, 3}} \tilde{d}_{k, m r}^{2}+\frac{1}{2 \eta_{k, 4}} \tilde{d}_{k, m l}^{2}\right],
\end{aligned}
$$

where $\eta_{k, 1}>0, \eta_{k, 2}>0, \quad \eta_{k, 3}>0$, and $\eta_{k, 4}>0$ are design parameters.

Now, we can have the time derivative of $V_{m_{k}}$ as follows:

$$
\begin{aligned}
& \dot{V}_{m_{k}}=\dot{V}_{m_{k}-1}+\sum_{k=1}^{M}\left(z_{k, m_{k}} \dot{z}_{k, m_{k}}+\frac{1}{\eta_{k, 1}} \widetilde{m}_{k, r} \dot{\tilde{m}}_{k, r}+\frac{1}{\eta_{k, 2}} \tilde{m}_{k, l} \dot{\tilde{m}}_{k, l}+\frac{1}{\eta_{k, 3}} \tilde{d}_{k, m r} \dot{\tilde{d}}_{k, m r}+\frac{1}{\eta_{k, 4}} \tilde{d}_{k, m l} \dot{\tilde{d}}_{k, m l}\right) \\
& =\dot{V}_{m_{k}-1}+\sum_{k-1}^{M}\left[z_{k, m_{k}}\left(u_{k, d}+\left(\tilde{d}_{k, m r}-\frac{u_{k, d}+\widehat{d}_{k, m r}}{\hat{m}_{k, r}} \widetilde{m}_{k, r}\right) \delta_{k}-\left(\tilde{d}_{k, m l}+\frac{u_{k, d}-\widehat{d}_{k, m l}}{\hat{m}_{k, l}} \widetilde{m}_{k, l}\right)\left(1-\delta_{k}\right)+\varepsilon_{k, d}-\ell_{k, m_{k}}\left(\widehat{x}_{k, 1}-y_{k}\right)-\dot{\omega}_{k, m_{k}-1}\right)\right. \\
& \left.+\frac{1}{\eta_{k, 1}} \tilde{m}_{k, r} \dot{\tilde{m}}_{k, r}+\frac{1}{\eta_{k, 2}} \widetilde{m}_{k, l} \dot{\vec{m}}_{k, l}+\frac{1}{\eta_{k, 3}} \tilde{d}_{k, m r} \dot{\tilde{d}}_{k, m r}+\frac{1}{\eta_{k, 4}} \tilde{d}_{k, m l} \dot{\tilde{d}}_{k, m l}\right] \\
& =\dot{V}_{m_{k}-1}+\sum_{k-1}^{M}\left[\begin{array}{c}
z_{k, m_{k}}\left(u_{k, d}+\varepsilon_{k, d}-\ell_{k, m_{k}}\left(\widehat{x}_{k, 1}-y_{k}\right)-\dot{\omega}_{k, m_{k}-1}\right)+\frac{1}{\eta_{k, 1}} \widetilde{m}_{k, r}\left(\dot{\tilde{m}}_{k, r}-\frac{u_{k, d}+\widehat{d}_{k, m r}}{\hat{m}_{k, r}} z_{k, m_{k}} \eta_{k, 1} \delta_{k}\right)+\frac{1}{\eta_{k, 2}} \widetilde{m}_{k, l}\left(\dot{\tilde{m}}_{k, l}-\frac{u_{k, d}-\hat{d}_{k, m l}}{\hat{m}_{k, l}} z_{k, m_{k}} \eta_{k, 2}\left(1-\delta_{k}\right)\right) \\
+\frac{1}{\eta_{k, 3}} \tilde{d}_{k, m r}\left(\dot{\tilde{d}}_{k, m r}+z_{k, m_{k}} \eta_{k, 3} \delta_{k}\right)+\frac{1}{\eta_{k, 4}} \tilde{d}_{k, m l}\left(\dot{\tilde{d}}_{k, m l}-z_{k, m_{k}} \eta_{k, 4}\left(1-\delta_{k}\right)\right)
\end{array}\right]
\end{aligned}
$$

Because

$$
\text { we can obtain }
$$

$$
z_{k, m_{k}} \varepsilon_{k, d} \leq z_{k, m_{k}}^{2}+\frac{1}{4} \varepsilon_{k, d}^{2}
$$

$\dot{V}_{m_{k}} \leq \dot{V}_{m_{k}-1}+\sum_{k=1}^{M}\left[\begin{array}{c}z_{k, m_{k}}\left(u_{k, d}+z_{k, m_{k}}-\ell_{k, m_{k}}\left(\hat{x}_{k, 1}-y_{k}\right)-\dot{\omega}_{k, m_{k}-1}\right)+\frac{1}{\eta_{k, 1}} \tilde{m}_{k, r}\left(\dot{\tilde{m}}_{k, r}-\frac{u_{k, d}+\hat{d}_{k, m r}}{\hat{m}_{k, r}} z_{k, m_{k}} \eta_{k, 1} \delta_{k}\right)+\frac{1}{\eta_{k, 2}} \tilde{m}_{k, l}\left(\dot{\tilde{m}}_{k, l}-\frac{u_{k, d}-\hat{d}_{k, m l}}{\hat{m}_{k, l}} z_{k, m_{k}} \eta_{k, 2}\left(1-\delta_{k}\right)\right)+\frac{1}{\eta_{k, 3}} \tilde{d}_{k, m r}\left(\dot{\tilde{d}}_{k, m r}+z_{k, m_{k}} \eta_{k, 3} \delta_{k}\right) \\ +\frac{1}{\eta_{k, 4}} \tilde{d}_{k, m l}\left(\dot{\dot{d}}_{k, m l}-z_{k, m_{k}} \eta_{k, 4}\left(1-\delta_{k}\right)\right)+\frac{1}{4} \varepsilon_{k, d}^{2}\end{array}\right]$.

Design $u_{k, d}, \widehat{m}_{k, r}, \widehat{m}_{k, l}, \widehat{d}_{k, m r}$, and $\widehat{d}_{k, m l}$ as follows:

$$
\begin{gathered}
u_{k, d}=-c_{k, m_{k}} z_{k, m_{k}}-z_{k, m_{k}-1}+\ell_{k, m_{k}}\left(\widehat{x}_{k, 1}-y_{k}\right) \\
-\frac{\omega_{k, m_{k}-1}-\alpha_{k, m_{k}-1}}{v_{k, m_{k}-1}}, \\
\dot{\hat{m}}_{k, r}=\frac{u_{k, d}+\widehat{d}_{k, m r}}{\widehat{m}_{k, r}} z_{k, m_{k}} \eta_{k, 1} \delta_{k}+2 \mu_{k, 1} \widehat{m}_{k, r},
\end{gathered}
$$

$$
\begin{aligned}
& \dot{\hat{m}}_{k, l}=\frac{u_{k, d}-\hat{d}_{k, m l}}{\hat{m}_{k, l}} z_{k, m_{k}} \eta_{k, 2}\left(1-\delta_{k}\right)+2 \mu_{k, 2} \widehat{m}_{k, l}, \\
& \dot{\hat{d}}_{k, m r}=-z_{k, m_{k}} \eta_{k, 3} \delta_{k}+2 \mu_{k, 3} \widehat{d}_{k, m r}, \\
& \dot{\hat{d}}_{k, m l}=z_{k, m_{k}} \eta_{k, 4}\left(1-\delta_{k}\right)+2 \mu_{k, 4} \widehat{d}_{k, m l},
\end{aligned}
$$

where $c_{k, m_{k}}>0, \mu_{k, 1}>0, \mu_{k, 2}>0, \mu_{k, 3}>0$, and $\mu_{k, 4}>0$ are design parameters.

Substituting (63)-(67) into (62) results in 


$$
\begin{aligned}
\dot{V}_{m_{k}} \leq & \sum_{k=1}^{M}\left\{-q_{k, 1}\left\|\underline{\tilde{x}}_{k}\right\|^{2}\right\}+\sum_{k=1}^{M}\left[-\sum_{i=1}^{m_{k}}\left(c_{k, i}-1\right) z_{k, i}^{2}+\sum_{i=1}^{m_{k}-1}\left(\frac{5}{4}-\frac{1}{v_{k, i}}\right) e_{k, i}^{2}-\sum_{i=1}^{2} \frac{\sigma_{k, i}}{2 \gamma_{k, i}} \widetilde{\omega}_{k, i}^{2}+\sum_{i=1}^{2} \frac{2 \sigma_{k, i}}{\gamma_{k, i}} \varpi_{k, i}^{* 2}\right. \\
& \left.+\sum_{i=1}^{m_{k}-1} \frac{1}{4} \psi_{k, i}^{2}+d_{k, 1}+\frac{1}{4} \varepsilon_{k, d}^{2}+\frac{2 \mu_{k, 1}}{\eta_{k, 1}} \widetilde{m}_{k, r} \widehat{m}_{k, r}+\frac{2 \mu_{k, 2}}{\eta_{k, 2}} \widetilde{m}_{k, l} \widehat{m}_{k, l}+\frac{2 \mu_{k, 3}}{\eta_{k, 3}} \widetilde{d}_{k, m r} \widehat{d}_{k, m r}+\frac{2 \mu_{k, 4}}{\eta_{k, 4}} \widetilde{d}_{k, m l} \widehat{d}_{k, m l}\right]
\end{aligned}
$$

Define

$$
\begin{aligned}
H= & \sum_{i=1}^{2} \frac{\sigma_{k, i}}{\gamma_{k, i}} \Phi_{k, i}^{* 2}+\frac{\mu_{k, 1}}{\eta_{k, 1}} m_{k, r}^{2}+\frac{\mu_{k, 2}}{\eta_{k, 2}} m_{k, l}^{2}+\frac{\mu_{k, 3}}{\eta_{k, 3}} d_{k, m r}^{2} \\
& +\frac{\mu_{k, 4}}{\eta_{k, 4}} d_{k, m l}^{2}+\sum_{i=1}^{m_{k}-1} \frac{1}{4} \psi_{k, i}^{2}+d_{k, 1}+\frac{1}{4} \bar{\varepsilon}_{k, d}^{2},
\end{aligned}
$$

where $\left|\varepsilon_{k, d}\right|<\bar{\varepsilon}_{k, d}$.

Substituting (69) into (68) leads to

$$
\begin{aligned}
\dot{V}_{m_{k}} \leq & \sum_{k=1}^{M}\left\{-q_{k, 1}\left\|\underline{\tilde{x}}_{k}\right\|^{2}\right\}+\sum_{k=1}^{M}\left[-\sum_{i=1}^{m_{k}}\left(c_{k, i}-1\right) z_{k, i}^{2}\right. \\
& -\sum_{i=1}^{m_{k}-1}\left(\frac{1}{v_{k, i}}-\frac{5}{4}\right) e_{k, i}^{2}-\sum_{i=1}^{2} \frac{\sigma_{k, i}}{2 \gamma_{k, i}} \widetilde{\Phi}_{k, i}^{2}-\frac{\mu_{k, 1}}{\eta_{k, 1}} \widetilde{m}_{k, r}^{2} \\
& \left.-\frac{\mu_{k, 2}}{\eta_{k, 2}} \widetilde{m}_{k, l}^{2}-\frac{\mu_{k, 3}}{\eta_{k, 3}} \widetilde{d}_{k, m r}^{2}-\frac{\mu_{k, 4}}{\eta_{k, 4}} \tilde{d}_{k, m l}^{2}+H\right] .
\end{aligned}
$$

\section{Stability Analysis}

Define Lyapunov function of the closed-loop system as $\widehat{V}=V_{m_{k}}$, and we can have $\widehat{V}=\dot{V}_{m_{k}}$. Let the design parameters satisfy

$$
\begin{aligned}
& c_{k, i}-1>0, \quad i=1,2, \ldots, m_{k}, \\
& \frac{1}{v_{k, i}}-\frac{5}{4}>0, \quad i=1,2, \ldots, m_{k}-1 .
\end{aligned}
$$

Define

$$
\begin{aligned}
C_{k}= & \min \left\{\frac{2 q_{k, 1}}{\lambda_{\max }\left(P_{k}\right)}, 2\left(c_{k, i}-1\right), 2 \sigma_{k, 1}, 2 \sigma_{k, 2},\right. \\
& \left.\cdot 2 \mu_{k, 1}, 2 \mu_{k, 2}, 2 \mu_{k, 3}, 2 \mu_{k, 4}, 2\left(\frac{1}{v_{k, i}}-\frac{5}{4}\right)\right\},
\end{aligned}
$$

where $i=1,2, \ldots, m_{k}$.

Define $C=\min _{k=1, \ldots, M}\left\{C_{k}\right\}$, and (70) can be rearranged as

$$
\widehat{V} \leq-C \widehat{V}+H
$$

Then, we have

$$
e^{C t} \dot{\vec{V}} \leq(-C \widehat{V}+H) e^{C t}
$$

$$
\begin{gathered}
\frac{\mathrm{d}}{\mathrm{d} t}\left(\widehat{V} e^{C t}\right) \leq H e^{C t}, \\
\widehat{V} e^{C t}-\widehat{V}(0) \leq \frac{H}{C}\left(e^{C t}-1\right), \\
0 \leq \widehat{V}(t) \leq \hat{V}(0) e^{-C t}+\frac{H}{C}\left(1-e^{-C t}\right) \leq \widehat{V}(0)+\frac{H}{C} .
\end{gathered}
$$

From (78), we can get that all the signals of the closed system, such as $x_{k, i}(t), \widehat{x}_{k, i}(t), z_{k, i}(t), a_{k, i}(t)$, and $u_{k}(t)$, are semiglobally uniformly ultimately bounded (SGUUB). Moreover, the observer error satisfies $\left\|\underline{\tilde{x}}_{k}\right\| \leq$ $\sqrt{\left(2 \hat{V}(0) e^{-C t}+(H / C)\right) / \lambda_{\min }\left(P_{k}\right)}$, and the tracking error satisfies $\left\|z_{k, 1}\right\| \leq \sqrt{2 \hat{V}(0) e^{-C t}+(H / C)}$.

Remark 3. Note that from (78), we can only conclude that the state observer errors and tracking errors do not converge to zero. However, we can make the state observer errors and tracking errors to be small by choosing design parameters.

Remark 4. It is worth mentioning that the influence of dead zone is compensated by inverse transformation technology, and dead zone parameters can be identified online, which is different from that in [17]. In [17], the problem of the dead zone is solved by constructing a tan-type BLF, which can compensate the influence of the dead zone but cannot get the dead zone parameters.

The configuration of the output feedback control scheme proposed above for the nonstrict feedback system is shown in Figure 1.

\section{Comparisons with Some Previous Results}

To further illustrate the contributions of this method, some comparisons with previous results in $[10-32,36,37]$ will be given in this section.

(1) The control methods in $[13,17,19-24]$ can address only nonlinear system (78) without switching signals:

$\left\{\begin{array}{l}\dot{x}_{i}=x_{i+1}+f_{i}\left(x_{1}, x_{2}, \ldots, x_{n}\right), \quad(i=1,2, \ldots, n-1), \\ \dot{x}_{n}=u(t)+f_{n}\left(x_{1}, x_{2}, \ldots, x_{n}\right), \\ y=x_{1} .\end{array}\right.$

The control methods in $[25-28,32]$ can only control switched systems (79), not large-scale systems: 


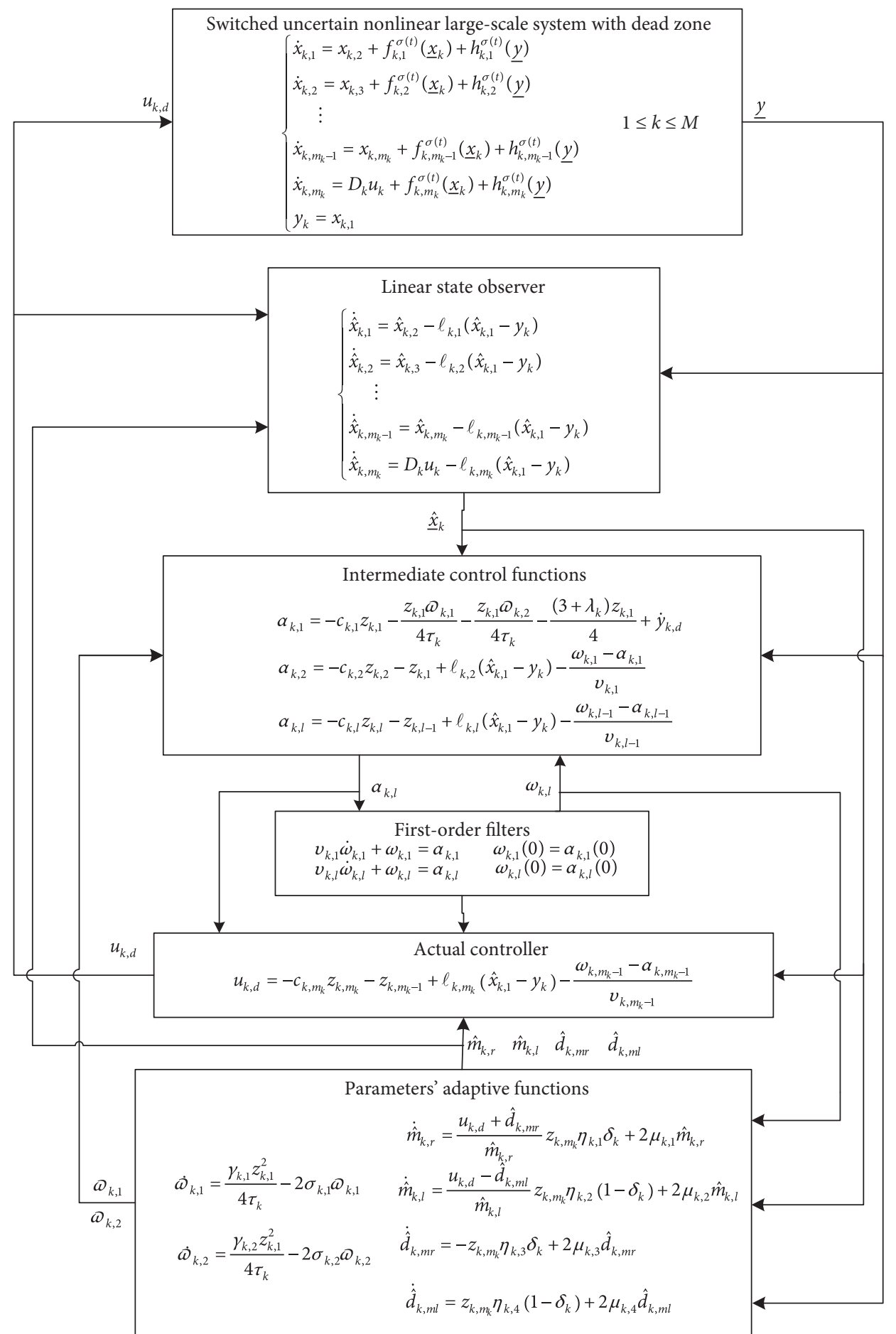

Figure 1: Block diagram of the control scheme.

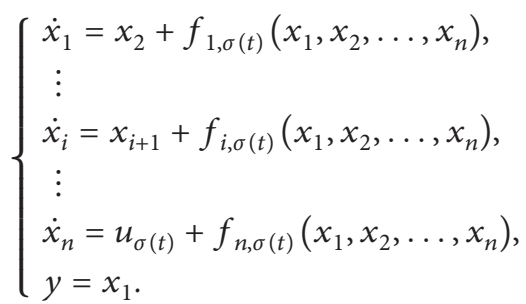

The control methods in $[14-16,18,29,36,37]$ address the following strict feedback system:

$$
\left\{\begin{array}{l}
\dot{x}_{i}=f_{i}\left(\bar{x}_{n}\right)+g_{i}\left(\bar{x}_{n}\right) x_{i+1}, \quad(i=1,2, \ldots, n-1), \\
\dot{x}_{n}=f_{n}\left(\bar{x}_{n}\right)+g_{n}\left(\bar{x}_{n}\right) u, \\
y=x_{1},
\end{array}\right.
$$


where $\bar{x}_{i}=\left[x_{1}, x_{2}, \ldots, x_{i}\right]^{T}$ and $f_{i}\left(\bar{x}_{i}\right)$ and $g_{i}\left(\bar{x}_{i}\right)$ are the functions of partial state variables.

The control method in this paper is designed for the nonlinear large-scale nonstrict feedback system (81) with arbitrary switching signals:

$$
\left\{\begin{array}{l}
\dot{x}_{k, 1}=x_{k, 2}+f_{k, 1}^{\sigma(t)}\left(\underline{x}_{k}\right)+h_{k, 1}^{\sigma(t)}(\underline{y}), \\
\dot{x}_{k, 2}=x_{k, 3}+f_{k, 2}^{\sigma(t)}\left(\underline{x}_{k}\right)+h_{k, 2}^{\sigma(t)}(\underline{y}), \\
\vdots \\
\dot{x}_{k, m_{k}-1}=x_{k, m_{k}}+f_{k, m_{k}-1}^{\sigma(t)}\left(\underline{x}_{k}\right)+h_{k, m_{k}-1}^{\sigma(t)}(\underline{y}), \\
\dot{x}_{k, m_{k}}=D_{k} u_{k}+f_{k, m_{k}}^{\sigma(t)}\left(\underline{x}_{k}\right)+h_{k, m_{k}}^{\sigma(t)}(\underline{y}), \\
y_{k}=x_{k, 1},
\end{array}\right.
$$

where $f_{k, l}^{\sigma(t)}(\cdot)$ is the function of whole state variables and $\sigma(t)$ is the arbitrary switching signal.

Whether it is controller design or stability proof, switched large-scale nonstrict feedback systems are far more difficult than systems (78)-(80). We were unable to use the control methods in $[13,17,19-24]$ to control the switched system due to the switching problem. The control methods in [25-28, 32] also cannot be used because of the interconnected influence between subsystems of the large-scale system. Moreover, when the controller of the nonstrict feedback switched system is designed by the control methods in [14-16, 18, 29, 36, 37], the virtual control signal and adaptive law of each subsystem are the functions of whole state variables. Consequently, the algebraic loop problem arises, which makes the controller design of a nonstrict feedback switched system very difficult. Therefore, the controller design method of the nonstrict feedback switched largescale system (81) considered in this paper is quite different from that of the controller design methods in $[13-29,32,36,37]$.

(2) $\mathrm{Li}$ and Tong and Younsi et al. [10, 11] proposed adaptive control methods for the nonstrict feedback switched large-scale system, but the input constraints were not considered. Though Wang et al. [12] presented the control schemes for a system with input constrained systems, all states of the system should be measurable. The strict limitation makes these control methods difficult to realize in practical applications. Therefore, control methods in [10-12] cannot be used to control a nonlinear system with unmeasurable state and input constraint that is discussed in this paper.

(3) This proposed adaptive control scheme does not need $n$-order differentiable and bounded conditions of the input signals and a monotonically increasing condition of unknown functions. However, these strict assumptions are common in the existing references [30, 31]. Moreover, by adopting dynamic surface technology and first-order filters, this control scheme can avoid the problem of the "explosion of complexity." Therefore, this control scheme not only conforms to engineering practice but also has a simple algorithm and requires a small number of calculations.

\section{Simulations}

Consider the following switched nonstrict feedback largescale system [10]:

$$
\begin{aligned}
& \left\{\begin{array}{l}
\dot{x}_{1,1}=x_{1,2}+f_{1,1}^{\sigma(t)}\left(x_{1,1}, x_{1,2}\right)+h_{1,1}^{\sigma(t)}\left(y_{1}, y_{2}\right), \\
\dot{x}_{1,2}=D_{1} u_{1}+f_{1,2}^{\sigma(t)}\left(x_{1,1}, x_{1,2}\right)+h_{1,2}^{\sigma(t)}\left(y_{1}, y_{2}\right), \\
y_{1}=x_{1,1},
\end{array}\right. \\
& \left\{\begin{array}{l}
\dot{x}_{2,1}=x_{2,2}+f_{2,1}^{\sigma(t)}\left(x_{2,1}, x_{2,2}\right)+h_{2,1}^{\sigma(t)}\left(y_{1}, y_{2}\right), \\
\dot{x}_{2,2}=D_{2} u_{2}+f_{2,2}^{\sigma(t)}\left(x_{2,1}, x_{2,2}\right)+h_{2,2}^{\sigma(t)}\left(y_{1}, y_{2}\right), \\
y_{2}=x_{2,1},
\end{array}\right.
\end{aligned}
$$

where $\sigma(t)=1,2, f_{1,1}^{1}\left(x_{1,1}, x_{1,2}\right)=x_{1,1}^{2}\left(1-\cos \left(x_{1,1} x_{1,2}\right)+\right.$ $\left.x_{1,2}\right)-0.2 x_{1,1} \sin \left(x_{1,1} x_{1,2}\right), \quad h_{1,1}^{1}\left(y_{1}, y_{2}\right)=y_{1} y_{2}, \quad f_{1,2}^{1}\left(x_{1,1}\right.$, $\left.x_{1,2}\right)=x_{1,1} x_{1,2}+x_{1,1}, h_{1,2}^{1}\left(y_{1}, y_{2}\right)=y_{1}^{2}+y_{2}^{2}, f_{2,1}^{1}\left(x_{2,1}, x_{2,2}\right)$ $=x_{2,1} \sin \left(x_{2,1} x_{2,2}\right)-0.5 x_{2,1} \sin \left(x_{2,1} x_{2,2}\right), \quad h_{2,1}^{1}\left(y_{1}, y_{2}\right)=y_{1}^{3}$ $y_{2}^{3}, \quad f_{2,2}^{1}\left(x_{2,1}, x_{2,2}\right)=x_{2,1} x_{2,2}, \quad h_{2,2}^{1}\left(y_{1}, y_{2}\right)=y_{1}-2 y_{2}, \quad f_{1,1}^{2}$ $\left(x_{1,1}, x_{1,2}\right)=x_{1,1}^{2}\left(1-\cos \left(x_{1,1} x_{1,2}\right)+x_{1,2}\right)-0.3 x_{1,1} \sin \left(x_{1,1}\right.$ $\left.x_{1,2}\right), h_{1,1}^{2}\left(y_{1}, y_{2}\right)=4 y_{1} y_{2}, f_{1,2}^{2}\left(x_{1,1}, x_{1,2}\right)=3 x_{1,1}, h_{1,2}^{2}\left(y_{1}\right.$, $\left.y_{2}\right)=y_{1}^{2}+2 y_{2}^{2}, \quad f_{2,1}^{2}\left(x_{2,1}, x_{2,2}\right)=\cos \left(x_{2,1} x_{2,2}\right), \quad h_{2,1}^{2}\left(y_{1}, y_{2}\right)$ $=3 y_{1}^{2} y_{2}^{2}, \quad f_{2,2}^{2}\left(x_{2,1}, x_{2,2}\right)=0.8 x_{2,1} x_{2,2}, \quad$ and $h_{2,2}^{2}\left(y_{1}, y_{2}\right)=$ $y_{1}-3 y_{2}$. The given tracking signals are $y_{1, d}=y_{2, d}=$ $0.25 \sin (t)+0.25 \sin (0.5 t)$.

Remark 5. Note that if system (82) does not contain the switching signal $\sigma(t)$, it turns into a nonlinear large-scale system, which was extensively studied in [17-22].

Remark 6. Note that functions $f_{k, l}^{\sigma(t)}\left(\underline{x}_{k}\right)$ in (82) do not satisfy the assumptions in $[28,29]$, i.e., we cannot find increasing smooth functions $\phi_{k, l}^{\sigma(t)}\left(\left\|\underline{x}_{k}\right\|\right)$ with $\phi_{k, l}^{\sigma(t)}(0)=0$, such that $f_{k, l}^{\sigma(t)}\left(\underline{x}_{k}\right) \leq \phi_{k, l}^{\sigma(t)}\left(\left\|\underline{x}_{k}\right\|\right)$. In addition, $(82)$ is not a switched strict feedback nonlinear system. Therefore, the control methods in [13-16, 27, 34, 35] cannot be used to control the system (82).

Choose the parameters as $\ell_{1,1}=\ell_{1,2}=\ell_{2,1}=\ell_{2,2}=10$, $c_{1,1}=40, \quad c_{1,2}=20, \quad c_{2,1}=20, \quad c_{2,2}=20, \quad \lambda_{1}=\lambda_{2}=1$, $\tau_{1}=\tau_{2}=1, \gamma_{1,1}=\gamma_{1,2}=1, \gamma_{2,1}=\gamma_{2,2}=2, \sigma_{1,1}=\sigma_{1,2}=0.05$, $\sigma_{2,1}=\sigma_{2,2}=0.1, \quad v_{1,1}=v_{1,2}=v_{2,1}=v_{2,2}=0.01, \quad m_{1, r}=2$, $m_{1, l}=1.3, \quad d_{1, r}=0.4, \quad d_{1, l}=0.6, \quad m_{2, r}=1.1, \quad m_{2, l}=2$, $d_{2, r}=0.2$, and $d_{2, l}=0.5$.

Remark 7. It is worth pointing out that the selected design parameters mentioned above are the only sufficient conditions for guaranteeing the stability of the control system. Of course, in the simulation, we can select another group of design parameters based on the selection guidelines of the design parameters.

The simulation results are shown in Figures 2-7, where Figure 2 shows the trajectories of the first subsystem's output $y_{1}$ and tracking signal $y_{1, d}$; Figure 3 shows the trajectory of the first subsystem's tracking error $z_{1,1}$; Figure 4 shows the 


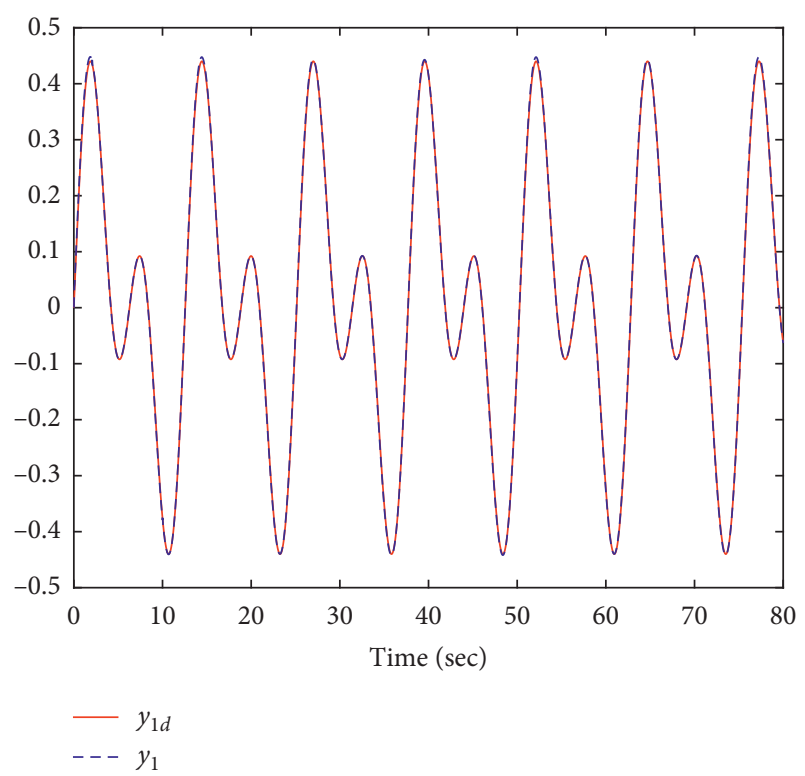

FIgURE 2: Trajectories of $y_{1}$ and $y_{1, d}$.

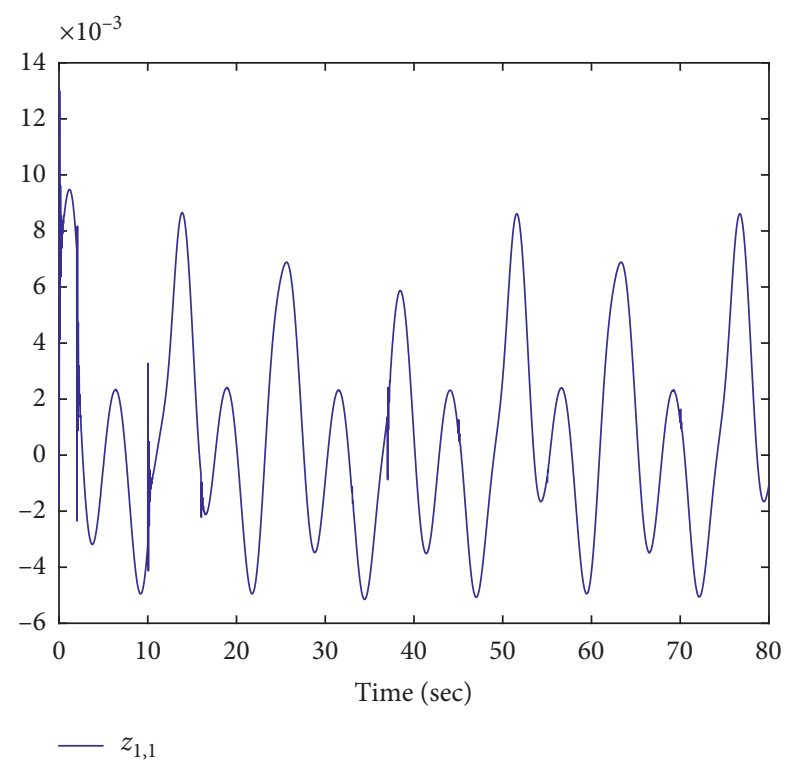

FIGURE 3: The tracking error $z_{1,1}$.

trajectories of the second subsystem's output $y_{2}$ and tracking signal $y_{2, d}$; Figure 5 shows the trajectory of the second subsystem's tracking error $z_{2,1}$; Figure 6 shows the trajectories of control signals $u_{k, d}$ and the output of dead zone $D_{k}\left(u_{k}\right)$; and Figure 7 shows the trajectory of the switched signal $\sigma(t)$.

From Figures 2, 3, and 7, we can see that a good tracking performance of the first subsystem is achieved under arbitrary switched signal and the absolute value of tracking error is less than 0.014. From Figures 4, 5, and 7, we can see that a

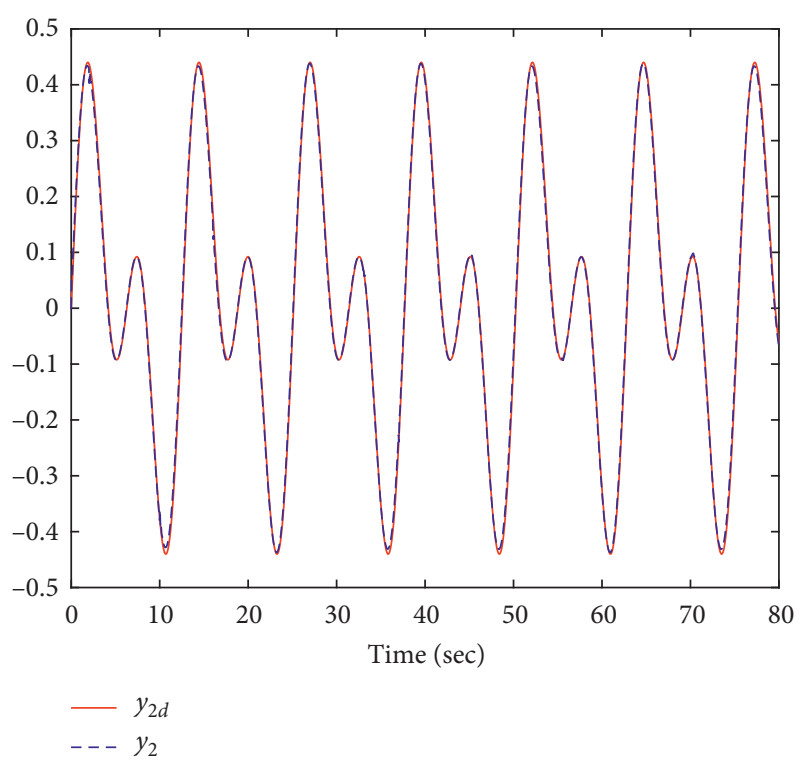

Figure 4: Trajectories of $y_{2}$ and $y_{2, d}$.

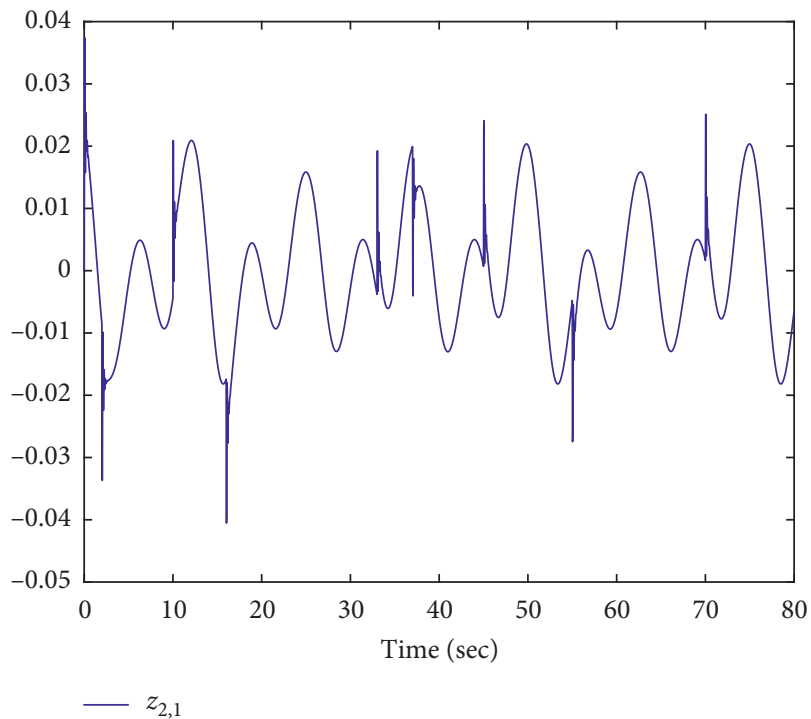

Figure 5: The tracking error $z_{2,1}$.

good tracking performance of the second subsystem is achieved under arbitrary switched signal and the absolute value of tracking error is less than 0.04. From Figure 6, we can see that the effect of unknown dead zone can be compensated well by the dead zone inverse technique.

From the simulation results, it can be seen that the proposed control scheme in this paper can guarantee the switched large-scale system to have a good tracking performance and can ensure the tracking errors to be kept in a small neighbourhood of zero. Moreover, all the signals of the closed-loop system are SGUUB. 

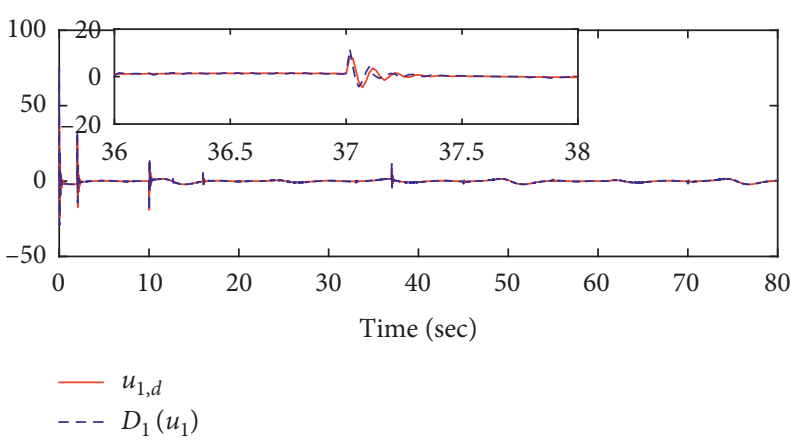

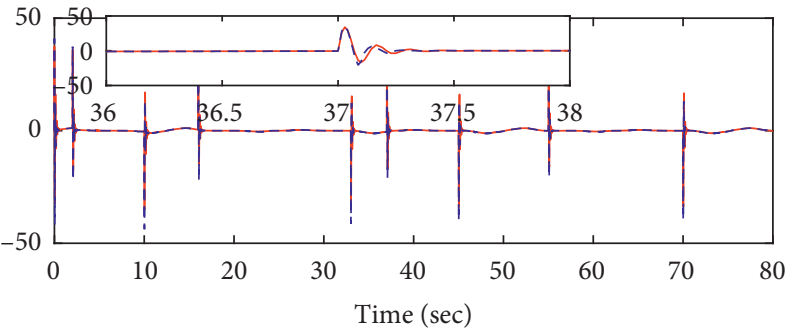

$-u_{2, d}$

$---D_{2}\left(u_{2}\right)$

(a)

(b)

FIgURE 6: Control inputs $u_{k, d}$ and $D_{k}\left(u_{k}\right)$.

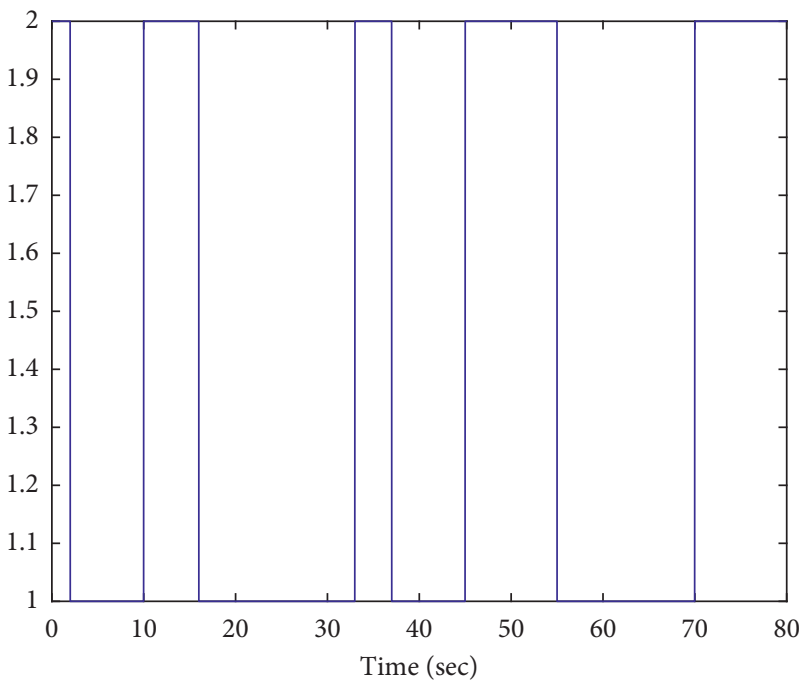

- Switching signal

FIgURE 7: The switched signal $\sigma(t)$.

\section{Conclusion}

In this paper, an adaptive fuzzy dynamic surface decentralized output feedback control scheme is proposed for large-scale interconnected uncertain nonstrict feedback switched systems. A decentralized linear state observer is designed to estimate the unmeasurable states of each subsystem. The dead zone problem is solved by using the dead zone inverse technology. The virtual control law, the real control law, and the adaptive law are designed by using the common Lyapunov function method to deal with the problem of arbitrary switching. Finally, the stability of the large-scale interconnected switched system is proved, and all the signals are guaranteed to be SGUUB. By choosing appropriate observer matrix parameters and controller design parameters, the tracking errors can be kept as small as possible. Future research directions are the extension of the results to stochastic nonlinear switched large-scale uncertain systems.

\section{Data Availability}

All the data included in this study are available from the corresponding author upon request.

\section{Conflicts of Interest}

The authors declare that there are no conflicts of interest regarding the publication of this paper.

\section{Acknowledgments}

This study was supported by the National Natural Science Foundation of China (51775463).

\section{References}

[1] S. Jain and F. Khorrami, "Decentralized adaptive control of a class of large-scale interconnected nonlinear systems," IEEE Transactions on Automatic Control, vol. 42, no. 2, pp. 136-154, 1997.

[2] K. Sun, S. Sui, and S. Tong, "Fuzzy adaptive decentralized optimal control for strict feedback nonlinear large-scale systems," IEEE Transactions on Cybernetics, vol. 48, no. 4, pp. 1326-1339, 2018.

[3] S. J. Yoo and J. B. Park, "Decentralized adaptive outputfeedback control for a class of nonlinear large-scale systems with unknown time-varying delayed interactions," Information Sciences, vol. 186, no. 1, pp. 222-238, 2012.

[4] H. Fan, L. Han, C. Wen, and L. Xu, "Decentralized adaptive output-feedback controller design for stochastic nonlinear interconnected systems," Automatica, vol. 48, no. 11, pp. 2866-2873, 2012.

[5] X. Li and X. Liu, "Backstepping-based decentralized adaptive neural Ho tracking control for a class of large-scale nonlinear interconnected systems," Journal of the Franklin Institute, vol. 355, no. 11, pp. 4533-4552, 2018.

[6] S. Sui, S. Tong, and Y. Li, "Observer-based adaptive fuzzy decentralized control for stochastic large-scale nonlinear systems with unknown dead-zones," Information Sciences, vol. 259, pp. 71-86, 2014.

[7] X. Zhang and Y. Lin, "Nonlinear decentralized control of large-scale systems with strong interconnections," Automatica, vol. 50, no. 9, pp. 2419-2423, 2014. 
[8] Y.-S. Huang and Z.-Y. Wang, "Decentralized adaptive fuzzy control for a class of large-scale MIMO nonlinear systems with strong interconnection and its application to automated highway systems," Information Sciences, vol. 274, pp. 210-224, 2014.

[9] T. Wang and S. Tong, "Decentralised output-feedback control design for switched fuzzy large-scale systems," International Journal of Systems Science, vol. 48, no. 1, pp. 171-181, 2017.

[10] Y. Li and S. Tong, "Fuzzy adaptive control design strategy of nonlinear switched large-scale systems," IEEE Transactions on Systems, Man, and Cybernetics: Systems, vol. 48, no. 12, pp. 2209-2218, 2018.

[11] L. E. Younsi, A. Benzaouia, and A. E. Hajjaji, "Decentralized control design for switching fuzzy large-scale T-S systems by switched Lyapunov function with $\$ \$ \mathrm{H}_{-}$linfty $\$ \$ \mathrm{H} \infty$ performance," International Journal of Fuzzy Systems, vol. 21, no. 4, pp. 1104-1116, 2019.

[12] L. Wang, C. L. P. Chen, and H. Li, "Event-triggered adaptive control of saturated nonlinear systems with time-varying partial state constraints," IEEE Transactions on Cybernetics, vol. 50, no. 4, pp. 1485-1497, 2020.

[13] L. Wang and C. L. P. Chen, "Reduced-order observer-based dynamic event-triggered adaptive NN control for stochastic nonlinear systems subject to unknown input saturation," IEEE Transactions on Neural Networks and Learning Systems, 2020.

[14] Q. Zhou, L. Wang, C. Wu, and H. Li, “Adaptive fuzzy tracking control for a class of pure-feedback nonlinear systems with time-varying delay and unknown dead zone," Fuzzy Sets and Systems, vol. 329, pp. 36-60, 2017.

[15] S. Tong and Y. Li, "Adaptive fuzzy output feedback tracking backstepping control of strict-feedback nonlinear systems with unknown dead zones," IEEE Transactions on Fuzzy Systems, vol. 20, no. 1, pp. 168-180, 2012.

[16] T. P. Zhang and S. S. Ge, "Adaptive dynamic surface control of nonlinear systems with unknown dead zone in pure feedback form," Automatica, vol. 44, no. 7, pp. 1895-1903, 2008.

[17] L. Wang and C. L. P. Chen, "Adaptive fuzzy dynamic surface control of nonlinear constrained systems with unknown virtual control coefficients," IEEE Transactions on Fuzzy Systems, vol. 28, no. 8, pp. 1737-1747, 2020.

[18] S. Tong, L. Zhang, and Y. Li, "Observed-based adaptive fuzzy decentralized tracking control for switched uncertain nonlinear large-scale systems with dead zones," IEEE Transactions on Systems, Man, and Cybernetics: Systems, vol. 46, no. 1, pp. 37-47, 2016.

[19] Y.-H. Liu, "Adaptive dynamic surface asymptotic tracking for a class of uncertain nonlinear systems," International Journal of Robust and Nonlinear Control, vol. 28, no. 4, pp. 1233-1245, 2018.

[20] Z. Lin, X. Liu, and Y. Li, “Adaptive fuzzy control for nonlinear pure-feedback systems with external disturbance and unknown dead zone output," International Journal of Fuzzy Systems, vol. 19, no. 6, pp. 1940-1949, 2017.

[21] H. Wang, P. Shi, H. Li, and Q. Zhou, "Adaptive neural tracking control for a class of nonlinear systems with dynamic uncertainties," IEEE Transactions on Cybernetics, vol. 47, no. 10, pp. 3075-3087, 2017.

[22] M. Wan and Q. Liu, "An improved adaptive fuzzy backstepping control for nonlinear mechanical systems with mismatched uncertainties," Automatika, vol. 60, no. 1, pp. 1-10, 2019.

[23] M. Wan, Q. Liu, J. Zheng, and J. Song, "Fuzzy state observerbased adaptive dynamic surface control of nonlinear systems with time-varying output constraints," Mathematical Problems in Engineering, vol. 2019, Article ID 3683581, 11 pages, 2019.

[24] M. Wan and Q. Liu, "Adaptive fuzzy backstepping control for uncertain nonlinear systems with tracking error constraints," Advances in Mechanical Engineering, vol. 11, no. 5, pp. 1-11, 2019.

[25] Y. Li and S. Tong, "Adaptive neural networks prescribed performance control design for switched interconnected uncertain nonlinear systems," IEEE Transactions on Neural Networks and Learning Systems, vol. 29, no. 7, pp. 3059-3068, 2017.

[26] L. Huang, Y. Li, and S. Tong, "Fuzzy adaptive output feedback control for a class of switched non-triangular structure nonlinear systems with time-varying delays," International Journal of Systems Science, vol. 49, no. 1, pp. 132-146, 2018.

[27] L. Etienne, K. M. D. Motchon, and S. Lecoeuche, "Stability analysis for switched uncertain nonlinear systems with dwell time and delay in the active mode detection," IEEE Control Systems Letters, vol. 3, no. 2, pp. 362-367, 2019.

[28] W. Wu, S. Tong, and Y. Li, "Fuzzy adaptive tracking control for switched nonlinear systems with full time-varying state constraints," Neurocomputing, vol. 352, 2019, https://doi.org/ 10.1016/j.neucom.2019.03.054.

[29] L. Zhang and G.-H. Yang, "Adaptive fuzzy output constrained decentralized control for switched nonlinear large-scale systems with unknown dead zones," Nonlinear Analysis: Hybrid Systems, vol. 23, pp. 61-75, 2017.

[30] B. Chen, C. Lin, X. Liu, and K. Liu, "Adaptive fuzzy tracking control for a class of MIMO nonlinear systems in nonstrictfeedback form," IEEE Transactions on Cybernetics, vol. 45, no. 12, pp. 2744-2755, 2015.

[31] H. Wang, X. Liu, K. Liu, and H. R. Karimi, "Approximationbased adaptive fuzzy tracking control for a class of nonstrictfeedback stochastic nonlinear time-delay systems," IEEE Transactions on Fuzzy Systems, vol. 23, no. 5, pp. 1746-1760, 2015.

[32] S. Tong, Y. Li, and S. Sui, "Adaptive fuzzy output feedback control for switched nonstrict-feedback nonlinear systems with input nonlinearities," IEEE Transactions on Fuzzy Systems, vol. 24, no. 6, pp. 1426-1440, 2016.

[33] S. Tong, Y. Li, and S. Sui, "Adaptive fuzzy tracking control design for SISO uncertain nonstrict feedback nonlinear systems," IEEE Transactions on Fuzzy Systems, vol. 24, no. 6, pp. 1441-1454, 2016.

[34] M. Wan, Q. Tian, and M. Wang, "Adaptive sliding mode control of robot manipulator based on second order approximation accuracy and decomposed fuzzy compensator," Wireless Personal Communications, vol. 103, no. 2, pp. 1207-1218, 2018.

[35] Z. Peng, J. Wang, and D. Wang, "Distributed maneuvering of autonomous surface vehicles based on neurodynamic optimization and fuzzy approximation," IEEE Transactions on Control Systems Technology, vol. 26, no. 3, pp. 1083-1090, 2018.

[36] Z. Peng, D. Wang, and J. Wang, "Predictor-based neural dynamic surface control for uncertain nonlinear systems in strict-feedback form," IEEE Transactions on Neural Networks and Learning Systems, vol. 9, no. 28, pp. 2156-2167, 2017.

[37] Z. Peng, J. Wang, and D. Wang, "Distributed containment maneuvering of multiple marine vessels via neurodynamicsbased output feedback," IEEE Transactions on Industrial Electronics, vol. 64, no. 5, pp. 3831-3839, 2017. 